\title{
Power Histories for Fuel Codes
}

Prepared by E. R. Gilbert, W. N. Rausch, F. E. Panisko

Pacific Northwest Laboratory

Operated by

Battelle Memorial Institute

Prepared for

U.S. Nuclear Regulatory

Commission 


\title{
NOTICE
}

This report was prepared as an account of work sponsored by an agency of the United States Government. Neither the United States Government nor any agency thereof, or any of their employees, makes any warranty, expressed or implied, or assumes any legal liability or responsibility for any third party's use, or the results of such use, of any information, apparatus product or process disclosed in this report, or represents that its use by such third party would not infringe privately owned rights.

\author{
Available from \\ GPO Sales Program \\ Division of Technical Information and Document Control \\ U. S. Nuclear Regulatory Commission \\ Washington, D. C. 20555 \\ Printed copy price: $\$ 3.25$ \\ and \\ Nätional Technical Information Service \\ Springfield, Virginia 22161
}




\section{Power Histories for Fuel Codes}

Manuscript Completed: October 1981

Date Published: January 1982

Prepared by

E. R. Gilbert, W. N. Rausch, F. E. Panisko

Pacific Northwest Laboratory

Richland, WA 99352

\section{Prepared for}

Division of Systems Integration

Office of Nuclear Reactor Regulation

U.S. Nuclear Regulatory Commission

Washington, D.C. 20555

NRC FIN B2343 



\section{ACKNOWLEDGMENTS}

The authors acknowledge the helpful discussions with colleagues in the Nuclear Fuels Section of the Materials Department at Pacific Northwest Laboratory. Especially valuable was the encouragement and guidance provided by J. C. Voglewede of the Core Performance Branch of the U.S. Nuclear Regulatory Comission. The computer-generated tables and figures in this report were prepared by W. D. Bennett, and the report was edited by S. K. Edier. 



\section{ABSTRACT}

Computations of power history effects on the pre-loss-of-coolant accident (LOCA) conditions of generic pressurized water reactor (PWR) and boiling water reactor (BWR) fuel rods were performed at Pacific Northwest Laboratory using the U.S. Nuclear Regulatory Commission (NRC) code FRAPCON-2. Comparisons were made between cases where the fuel operated at a high ("LOCA-limited") power throughout life $(20,000 \mathrm{MWd} / \mathrm{MTU})$ and those where the fuel was at a lower power for most of its burnup and ramped to the high power at 10,000 or 20,000 MWd/MTU burnup.

The PWR rod was calculated to have more cladding creepdown during the lower power cases, which resulted in slightly lower centerline temperatures (as much as $100^{\circ} \mathrm{C}$ ). This result was insensitive to the method used to increase the power during the ramps (i.e., by increasing the average rod power or by changing the peak-to-average (P/A) ratio of the axial power shape). The calculations also indicate that the highest fuel centerline temperatures were reached at startup.

The BWR rod, however, demonstrated a substantial dependence on the power history. In this case, the constant high-power rod released considerably more fission gas than the lower power cases (21\% versus $0.4 \%$ ), which resulted in temperature differences of up to $350^{\circ} \mathrm{C}$. The highest temperature was reached at end-of-life (EOL) in the constant high-power case. 



\section{SUMMARY}

The objective of this Pacific Northwest Laboratory (PNL) program was to assess the impact of some power history assumptions on end-of-life (EOL) fuel temperature calculations and recommend appropriate assumptions for safety analysis.

Two generic fuel rods were simulated with the U.S. Nuclear Regulatory Comission (NRC) audit code FRAPCON-2 to determine the effects of power history assumptions on pre-LOCA fuel temperatures. The fuel rods chosen for the study correspond to a commercial pressurized water reactor (PWR) $(15 \times 15)$ design and a commercial boiling water reactor $(B W R)(8 \times 8)$ design. The base case calculation for each rod was performed by extending the computer code to 20,000 MWd/MTU at the "loss-of-coolant accident (LOCA)-limited" linear heat generation rate (LHGR). (a) Computer runs were made to simulate the behavior of each fuel rod at 0.5 and 0.7 times the peak power. At various burnups, the code then modeled ramps to the LOCA-limited LHGR $(12.6 \mathrm{~kW} / \mathrm{ft}$ and $13.4 \mathrm{~kW} / \mathrm{ft}$ were chosen as typical values for the PWR and BWR rods, respectively). The PWR rods were ramped in two ways: by increasing the average power or by modifying the peak-to-average (P/A) ratio. The BWR rods were ramped by raising the average power to the LOCA-limited peak.

The PWR calculations gave very similar results regardless of the power history or method of ramping. The final temperature of the constant highpower case was higher than for the rods run at low powers and then ramped. This difference was less than $100^{\circ} \mathrm{C}$ and apparently resulted from increased cladding creepdown that was calculated to occur early in the life of the lowpower rods. The lower power rod experienced more creepdown than the higher power rod because fuel thermal expansion was less; thus, more cladding creepdown was allowed before the fuel supported the cladding.

(a) The highest power licensable due to considerations of potential cladding temperatures during a postulated LOCA. 
The BWR rod showed a definite power history effect. The most significant disparity between the high- and low-power cases occurred in the fission gas release fraction (21\% versus $0.4 \%$ ). Temperatures calculated in the low-power case ranged from $150^{\circ} \mathrm{C}$ at $10,000 \mathrm{MWd} / \mathrm{MTU}$ to $350^{\circ} \mathrm{C}$ at $20,000 \mathrm{MWd} / \mathrm{MTU}$ less than the temperatures calculated for the constant high-power case. 


\begin{tabular}{|c|c|c|c|c|c|c|c|c|c|c|c|c|c|c|}
\hline ACKNOWLED & GMENT & & . & . & . & . & . & - & . & - & . & - & - & $i i j$ \\
\hline ABSTRACT & . & . & . & . & . & . & . & . & • & $\cdot$ & , & - & $\cdot$ & $v$ \\
\hline SUMMARY & . & . & . & . & . & - & . & • & - & • & - & - & • & vit \\
\hline INTRDDUCT & ION & - & . & . & . & . & . & ${ }^{*}$ & $\cdot$ & • & • & - & - & 1 \\
\hline CONCLUSIO & & . & . & . & . & . & . & - & . & • & • & - & - & 2 \\
\hline ANALYSIS & $\mathrm{DF} P O$ & WER & HISTORY & & CTS & . & . & - & " & - & . & • & - & 3 \\
\hline APPR & $\mathrm{DACH}$ & . & . & . & . & . & . & $\cdot$ & • & • & • & - & • & 3 \\
\hline RE SU & LTS & . & . & . & . & . & . & • & . & - & . & • & • & o \\
\hline DISC & USSID & & . & . & . & . & . & • & • & - & • & - & • & 于 \\
\hline REFE RE NCE & & - & . & . & . & . & . & $\cdot$ & • & - & . & - & $\cdot$ & 13 \\
\hline APPENDIX & & . & . & . & . & . & . & . & . & . & . & - & . & A. \\
\hline
\end{tabular}





\section{FIGURES}

1 Calculated Effect of Power History on Pre-LOCA Fuel Centerline Temperature for a $15 \times 15$ PWR Rod with a Constant Peak-to-Average Power Ratio of 1.2 .

2 Calculated Effect of Power History on Pre-LOCA Fuel Centerline Temperature for a $8 \times 8$ BWR Rod with a Constant Peak-to-Average Power Ratio of 1.3 .

3 Calculated Effect of Power History on Pre-LOCA Fuel Centerline Temperature a for $15 \times 15$ PWR Rod with Constant Average Rod Power

4 Effect of Creepdown in the FRAPCON-2 Code When Compared with the Creepdown-Deficient GAPCON-2 Code for a

\section{TABLES}

1 Computer Code Input . . . . . . . . . .

A.1 FRAPCON-2 Prediction for $15 \times 15$ PWR with $12.6 \mathrm{~kW} / \mathrm{ft}$ to $20 \mathrm{GWd} / \mathrm{MTM}, \mathrm{P} / \mathrm{A}=1.2$

A.2 FRAPCON-2 Prediction for $8 \times 8$ BWR with $13.4 \mathrm{~kW} / \mathrm{ft}$ to $20 \mathrm{GW} / \mathrm{MTM}, \mathrm{P} / \mathrm{A}=1.3$.

A.3 FRAPCON-2 Prediction for $15 \times 15$ PWR with $6.3 \mathrm{~kW} / \mathrm{ft}$ for 1 Day, Ramp to $12.6 \mathrm{~kW} / \mathrm{ft}, P / A=1.2$

A.4 FRAPCON-2 Prediction for $15 \times 15 \mathrm{PWR}$ with $6.3 \mathrm{~kW} / \mathrm{ft}$ for $10 \mathrm{GWd} / \mathrm{MTM}$, Ramp to $12.6 \mathrm{~kW} / \mathrm{ft}, \mathrm{P} / \mathrm{A}=1.2 . \quad \cdot \quad \cdot \quad \cdot$

A.5 FRAPCON-2 Prediction for $15 \times 15$ PWR with $6.3 \mathrm{~kW} / \mathrm{ft}$ for $20 \mathrm{GWd} / \mathrm{MTM}$, Ramp to $12.6 \mathrm{~kW} / \mathrm{ft}, \mathrm{P} / \mathrm{A}=1.2 . \quad . \quad . \quad$.

A.6 FRAPCON-2 Prediction for $8 \times 8$ BWR with $6.7 \mathrm{~kW} / \mathrm{ft}$ for 1 Day, Ramp to $13.4 \mathrm{~kW} / \mathrm{ft}, \mathrm{P} / \mathrm{A}=1.3$ 
A.7 FRAPCON-2 Prediction for $8 \times 8$ BWR with $6.7 \mathrm{~kW} / \mathrm{ft}$ for $10 \mathrm{GWd} / \mathrm{MTM}$, Ramp to $13.4 \mathrm{~kW} / \mathrm{ft}, \mathrm{P} / \mathrm{A}=1.3$. . . . A.7

A. 8 FRAPCON-2 Prediction for $8 \times 8$ BWR with $6.7 \mathrm{~kW} / \mathrm{ft}$ for $20 \mathrm{GWd} / \mathrm{MTM}$, Ramp to $13.4 \mathrm{~kW} / \mathrm{ft}, \mathrm{P} / \mathrm{A}=1.3$. . . . . A.8

A.9 FRAPCON-2 Prediction for $15 \times 15$ PWR with $8.8 \mathrm{~kW} / \mathrm{ft}$ for 1 Day, Ramp to $12.6 \mathrm{~kW} / \mathrm{ft} P / A=1.2 . . . . . .$.

A.10 FRAPCON-2 Prediction for $15 \times 15 \mathrm{PWR}$ with $8.8 \mathrm{~kW} / \mathrm{ft}$ for 2 GWd $/$ MTM, Ramp to $12.6 \mathrm{~kW} / \mathrm{ft}, P / A=1.2 . . . \quad . \quad$. A.10

A.11 FRAPCON-2 Prediction for $15 \times 15 \mathrm{PWR}$ with $8.8 \mathrm{~kW} / \mathrm{ft}$ for $10 \mathrm{GWd} / \mathrm{MTM}$, Ramp to $12.6 \mathrm{~kW} / \mathrm{ft}, \mathrm{P} / \mathrm{A}=1.2 . . \quad . \quad$. $\mathrm{A} .11$

A.12 FRAPCON-2 Prediction for $15 \times 15$ PWR with $8.8 \mathrm{kw} / \mathrm{ft}$ for $20 \mathrm{GWd} / \mathrm{MTM}$, Ramp to $12.6 \mathrm{~kW} / \mathrm{ft}, \mathrm{P} / \mathrm{A}=1.2$. . . . . A.12

A.13 FRAPCON-2 Prediction for $8 \times 8$ BWR with $9.4 \mathrm{~kW} / \mathrm{ft}$ for 1 Oay,

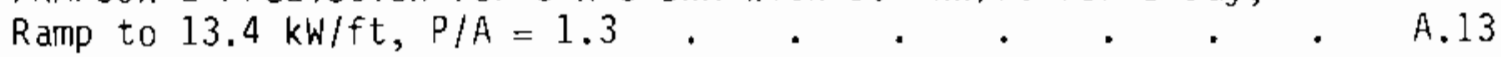

A.14 FRAPCON-2 Prediction for $8 \times 8$ BWR with $9.4 \mathrm{~kW} / \mathrm{ft}$ for $10 \mathrm{GWd} / \mathrm{MTM}$, Ramp to $13.4 \mathrm{~kW} / \mathrm{ft}, \mathrm{P} / \mathrm{A}=1.3$. . . . A.14

A.15 FRAPCON-2 Prediction for $8 \times 8$ BWR with $9.4 \mathrm{~kW} / \mathrm{ft}$ for $20 \mathrm{GWd} / \mathrm{MTM}$, Ramp to $13.4 \mathrm{~kW} / \mathrm{ft}, \mathrm{P} / \mathrm{A}=1.3$. . . . . A.15

A.16 FRAPCON-2 Prediction for $15 \times 15$ PWR with $8.8 \mathrm{~kW} / \mathrm{ft}$ for 1 Day, Ramp to $12.6 \mathrm{~kW} / \mathrm{ft}$, Constant Rod Average Power . . . . A.16

A.17 FRAPCON-2 Prediction for $15 \times 15$ PWR with $8.8 \mathrm{~kW} / \mathrm{ft}$ for $10 \mathrm{GWd} / \mathrm{MTM}$, Ramp to $12.6 \mathrm{~kW} / \mathrm{ft}$, Constant Rod Average Power . A.17

A.18 FRAPCON-2 Prediction for $15 \times 15 \mathrm{PWR}$ with $8.8 \mathrm{~kW} / \mathrm{ft}$ for $20 \mathrm{GWd} / \mathrm{MTM}$, Ramp to $12.6 \mathrm{~kW} / \mathrm{ft}$, Constant Rod Average Power . A.18

A.19 GAPCON-2 Prediction for $15 \times 15$ with $12.6 \mathrm{~kW} / \mathrm{ft}$ to

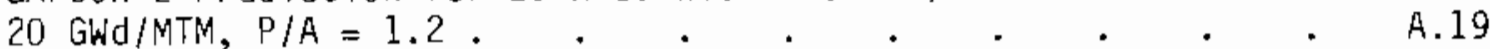

A.20 GAPCON-2 Prediction for $15 \times 15$ with $8.8 \mathrm{kw} / \mathrm{ft}$ for $20 \mathrm{GWd} / \mathrm{MTM}$ Ramp to $12.6 \mathrm{~kW} / \mathrm{ft}, P / \mathrm{A}=1.2$. 
INTRODUCTION

Steady-state fuel performance codes like FRAPCON-2, (1) a U.S. Nuclear Regulatory Commission (NRC) audit code, have their major impact on loss-ofcoolant accident (LOCA) analysis. Fuel rod temperatures, which are calculated as the starting point for the emergency core cooling system (ECCS) analysis, have a dominant effect on LOCA peak cladding temperature and are subject to large variations due to power history effects. The power history assumptions vary from vendor to vendor; therefore, there is a need to review some of the vendor assumptions to confirm their adequacy.

In reviewing safety analyses as part of the licensing of nuclear power plants, the NRC performs calculations of fuel parameters and response using fuel performance computer codes. The predictions of these codes are dependent on not only the conditions at the time of a hypothetical accident but also the conditions prior to the event. The purpose of this Pacific Northwest Laboratory $(\mathrm{PNL})^{(\mathrm{a})}$ program was to provide assistance in determining power history effects in fuel performance codes used for plant safety analyses.

Generic pressurized water reactor (PWR) and boiling water reactor (BWR) fuel rods were simulated using FRAPCON-2 to determine the effects of certain power history assumptions on pre-LOCA fuel temperatures. The base case caTculation for each rod was performed by extending the computer code to 20,000 MWd/MTU at the LOCA-limited linear heat generation rate (LHGR). Computer runs simulated the behavior of each fuel rod at 0.5 and 0.7 times the peak power. At various burnups, the calculations then modeled ramps to the LOCA-1 imited LHGR.

The remainder of this report consists of the conclusions that were reached and a presentation of the approach that was used, the results that were obtained, and a discussion of those results. The appendix contains power history data and code output for the generic PWR and BWR rods.

(a) Operated for the U.S. Department of Energy (DOE) by Battelle Memorial Institute. 


\section{CONCLUSIONS}

The results calculated by FRAPCON-2 showed that the specific choices of power history had very little effect on the EOL centerline temperatures for the PWR cases. In all PWR cases, the fuel rod was predicted to reach its highest temperature at beginning of life $(\mathrm{BOL})$. The method of ramping the fuel rod-changing the peak-to-average (P/A) ratio or increasing the average power--did not influence the results.

The BWR cases, however, demonstrated a significant dependence upon power history. The constant high-power rod released considerably more fission gas than the lower power cases; this resulted in EOL temperatures that were as

much as $350^{\circ} \mathrm{C}$ higher for the constant high-power case.

It appears that in the case of PWR fuel the method of modeling fuel performance by operating the fuel at typical commercial powers (6 to $9 \mathrm{~kW} / \mathrm{ft}$ ) and then ramping to the peak licensable LHGR may be a suitable substitute for calculating fuel thermal performance by modeling a constant high-power history. For the BWR, however, the constant high-power history case was more conservative at higher burnup conditions than the low-power/ramp technique. Aithough this does not necessarily invalidate the low-power method, it does indicate that more work would be necessary to assess which power history is more realistic with respect to licensing for modeling comercial fuel performance. 


\section{ANALYSIS OF POWER HISTORY EFFECTS}

This section includes a description of the approach that was used in the analysis, a presentation of the results, and a discussion of those results.

\section{APPROACH}

Representative power histories consisted of operation at a fixed power level followed by an instantaneous increase to a LOCA-limited peak power at which critical output parameters (e.g., volume average fuel temperature, rod internal pressure) were calculated. The calculational matrix included two fuel designs--a PWR $15 \times 15$ and a BWR $8 \times 8$ design(a)--three steady-state power levels--0.5, 0.7, and 1.0 times the fuel rod LOCA-limited peak power level. The power was increased by changing the rod average power while maintaining a constant P/A power ratio for both PWR and BWR cases. An additional set of cases was produced by changing the axial power distribution for some special PWR cases.

The specific cases used to assess the impact of power history assumptions on LOCA-limited fuel temperature cladding calculations were as follows: The power history effects on pre-LOCA conditions were computed for a generic $15 x$ $15 \mathrm{PWR}$ fuel rod with a peak LHGR of $12.6 \mathrm{~kW} / \mathrm{ft}^{(\mathrm{b})}$ and a steady-state pre-LOCA LHGR of $8.8 \mathrm{~kW} / \mathrm{ft}(0.7$ times the peak LHGR) and $6.3 \mathrm{~kW} / \mathrm{ft}(0.5 \mathrm{times}$ the peak LHGR) for burnups of $26 \mathrm{MWd} / \mathrm{MTU}$ (i.e., BOL), 10,000 MWd/MTU, and 20,000 MWd/MTU using the FRAPCON-2 code with FRACAS-2 mechanical model and the ANS 5.4 fission gas release model. A special case was conducted with a steady-state pre-LOCA LHGR of $8.8 \mathrm{~kW} / \mathrm{ft}$ for a burnup of $2000 \mathrm{MWd} / \mathrm{MTU}$. In all cases, the power was ramped to $12.6 \mathrm{~kW} / \mathrm{ft}$ at the specified burnup. The computer outputs are tabulated in Appendix A.

Similar computations were conducted for a generic $8 \times 8$ BWR fuel rod with a peak LHGR of $13.4 \mathrm{~kW} / \mathrm{ft}^{(\mathrm{b})}$ and a steady-state pre-LOCA LHGR of $9.4 \mathrm{~kW} / \mathrm{ft}$ (0.7 times the peak LHGR) and $6.7 \mathrm{~kW} / \mathrm{ft}(0.5$ times the peak LHGR) for burnups of BOL, 10,000 MWd/MTU, and 20,000 MWd/MTU.

(a) See Reference 2 for physical descriptions of the fuel rods.

(b) Peak power values were chosen to represent typical PWR and BWR LOCA-limited LHGRs. 
Computations were made for conditions of constant $P / A$ power ratios of 1.2 (PWR) and 1.3 (BWR). The power was increased at the specified burnup by increasing the rod power and maintaining the same $P / A$ power ratios. An alternate method of power ramping to LOCA conditions--maintaining a constant rod average power but altering the axial power distribution to enable attainment of the LOCA conditions in a segment of the rod--was performed for PWR burnups at BOL, 10,000 MWd/MTU, and 20,000 MWd/MTU with pre-LOCA power of $8.8 \mathrm{~kW} / \mathrm{ft}$ (0.7 times the peak LHGR). The power histories for these cases are also described in the appendix.

The FRAPCON-2 code, which is described in Reference 1 , is a steady-state model developed jointly by EG\&G Idaho ${ }^{(a)}$ and PNL for the Fuel Behavior Research Branch of the NRC. The FRACAS-2 mechanical subcode was selected to compute the fuel-cladding interactions, while fission gas release was computed with the ANS 5.4 option. FRACAS-2 uses the effective fuel conductivity and the relocated fuel-cladding gap size for thermal calculations. The relocated fuel surface is used in the mechanics calculations. A model is included for time-dependent hot pressing of misaligned fuel fragments. The FRAPCON-2 code includes cladding creepdown under the external pressure of the coolant. Code verification is presented in References 3 and 4 . The selection of code options as described in Reference 1 for these computations is presented in Table 1.

(a) Idaho National Engineering Laboratory is operated for DDE by EG\&G Idaho, Inc. 


\section{TABLE 1. Computer Code Input}
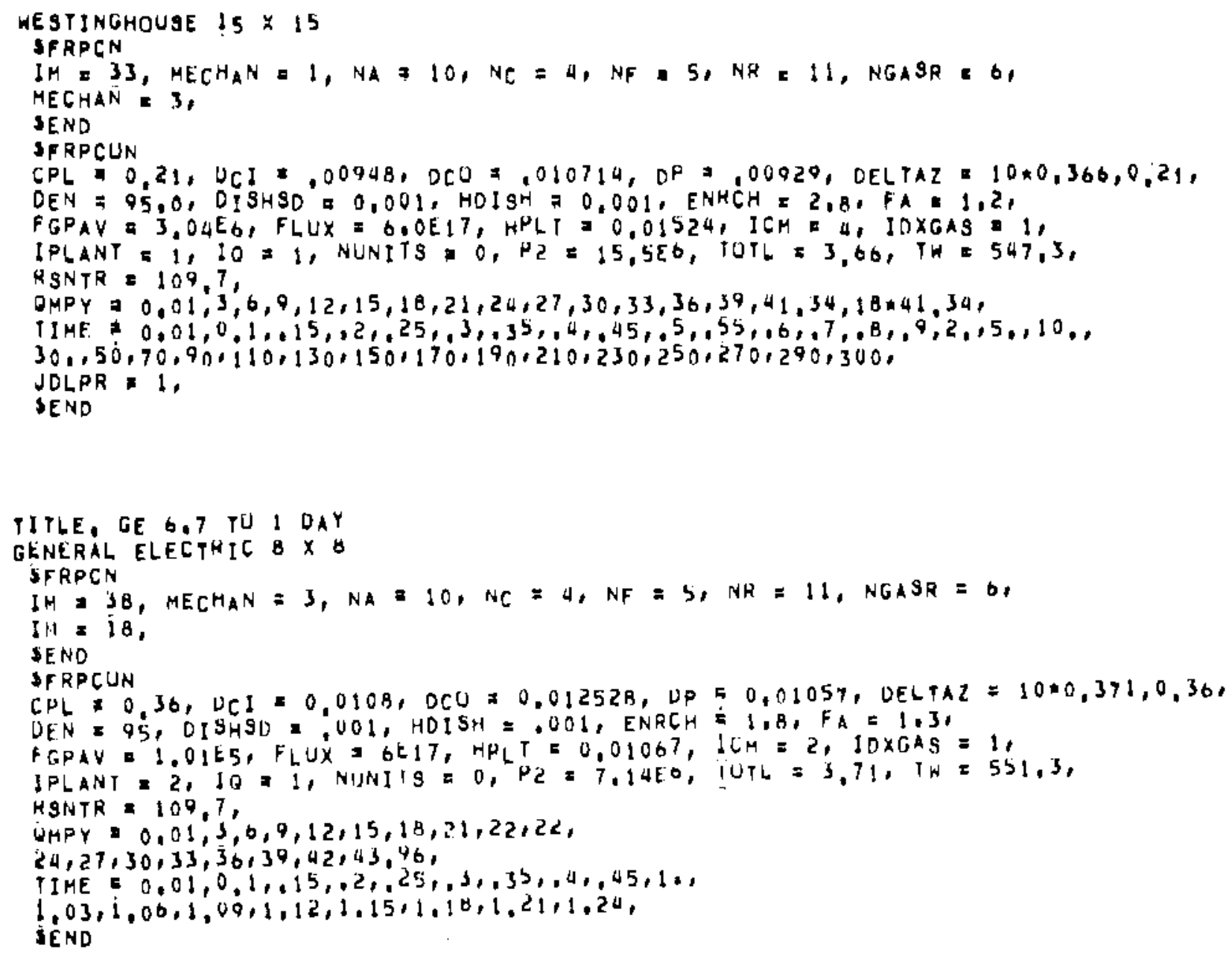
RESULTS

The results of the base test matrix for both PWR and BWR generic cases are listed in the Appendix (Tables A.1 through A.15) for constant P/A power ratios, and the results for the $15 \times 15$ PWR cases are plotted in Figure 1 . The power ramps executed at BOL from the 0.5 pre-LOCA power level ended precisely on the 1.0 power level curve and passed through the 0.7 level curve during the $r$ amp. The 0.7 case also ended precisely on the 1.0 level curve.

At $2000 \mathrm{MWd} / \mathrm{MTU}$, the 0.7 case fell short of reaching the 1.0 level curve by approximately $25^{\circ} \mathrm{C}$. At $10,000 \mathrm{MWd} / \mathrm{MTU}$, the 0.5 case fell short of reaching the 0.7 level curve during the transient by approximately $15^{\circ} \mathrm{C}$ and fell short of reaching the 1.0 level curve at the end of the transient by approximately $100^{\circ} \mathrm{C}$. The 0.7 case fell short of reaching the 1.0 case by approximately $45^{\circ} \mathrm{C}$. At $20,000 \mathrm{MWd} / \mathrm{MTU}$, the $0.5 \mathrm{case}$ fell short of reaching the 0.7 level curve during the transient by approximately $10^{\circ} \mathrm{C}$ and fell short of reaching the 1.0 level curve at the end of the transient by approximately $95^{\circ} \mathrm{C}$. The 0.7 case fell short of reaching the 1.0 level curve at the end of the transient by approximately $50^{\circ} \mathrm{C}$. The fuel centerline temperatures in Figure 1 were predicted to decrease by 100 to $150^{\circ} \mathrm{C}$ within the first $10,000 \mathrm{MWd} / \mathrm{MTJ}$ and then to stabilize during the second half of the computational period ito $20,000 \mathrm{MWd} / \mathrm{MTU})$.

The results for the $8 \times 8$ BWR cases are plotted in Figure 2 . The 0.5 case at BOL passed through the 0.7 curve during the ramp to the 1.0 level curve with which it agreed precisely. The 0.7 case agreed precisely with the 1.0 curve after being ramped at $80 \mathrm{~L}$. At $10,000 \mathrm{MWd} / \mathrm{MTU}$, the $0.5 \mathrm{case}$ fell short of the 0.7 curve by $50^{\circ} \mathrm{C}$ as it was being ramped to the 1.0 curve where it fell short by $190^{\circ} \mathrm{C}$. The ramped 0.7 case fell short of the 1.0 curve by $100^{\circ} \mathrm{C}$. At $20,000 \mathrm{MWd} / \mathrm{MTU}$, the 0.5 case fell $60^{\circ} \mathrm{C}$ short of the 0.7 curve as it was being ramped to the 1.0 level where it fell short by $390^{\circ} \mathrm{C}$. The 0.7 case fell short by $280^{\circ} \mathrm{C}$ of the 1.0 curve at the end of its ramp. The fuel centerline temperatures in Figure 2 were predicted to decrease by 100 to $140^{\circ} \mathrm{C}$ within the first $10,000 \mathrm{MWd} / \mathrm{MTU}$ and then to stabilize during the second half of the computational period, except for the steady-state case, at $13.4 \mathrm{~kW} / \mathrm{ft}$. In this case the centerline temperature increased by $200^{\circ} \mathrm{C}$ during the second half of the computational period. 


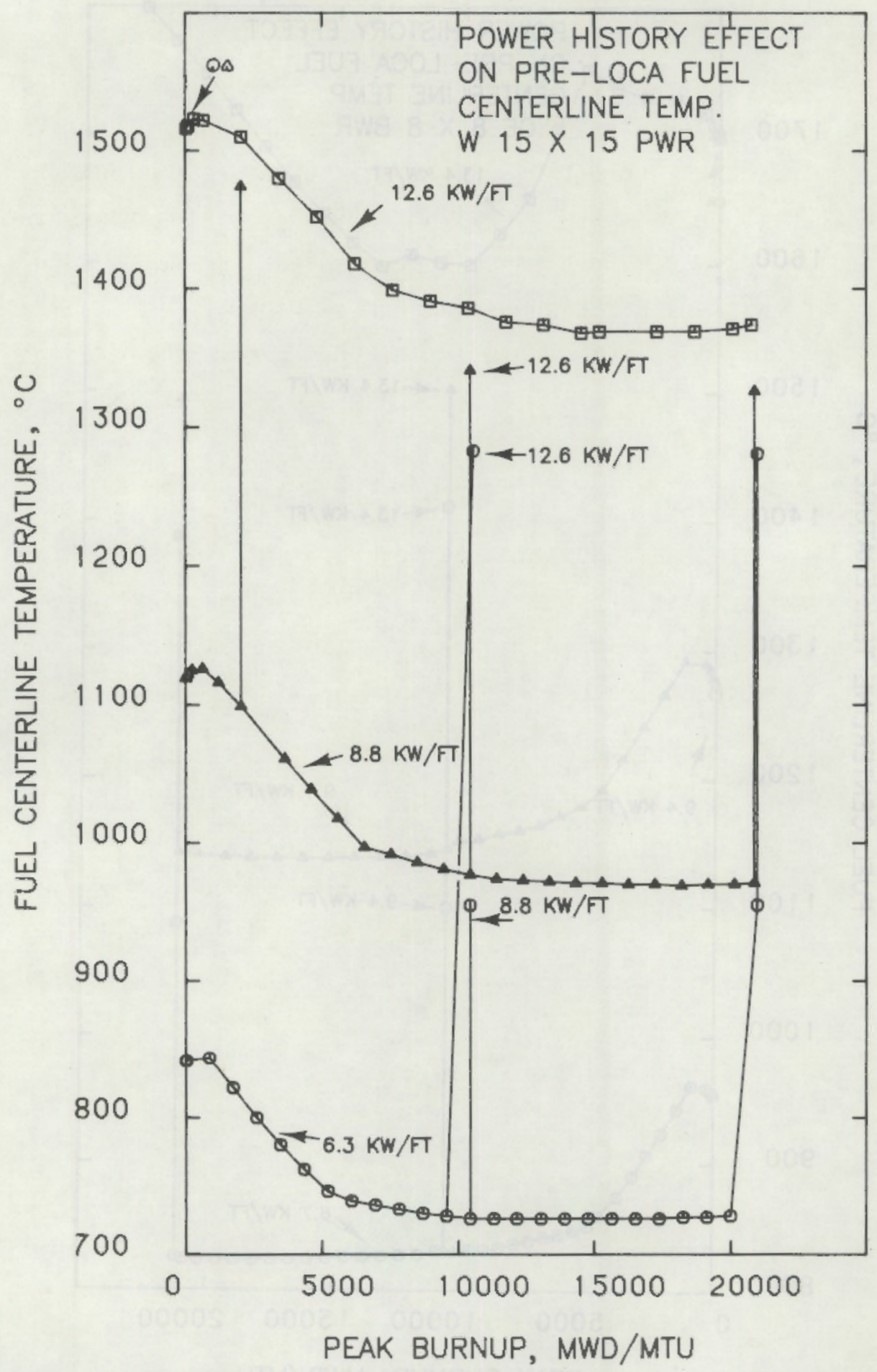

FIGURE 1. Calculated Effect of Power History on Pre-LOCA Fuel Centerline Temperature for a $15 \times 15$ PWR Rod with a Constant Peak-toAverage Power Ratio of 1.2 


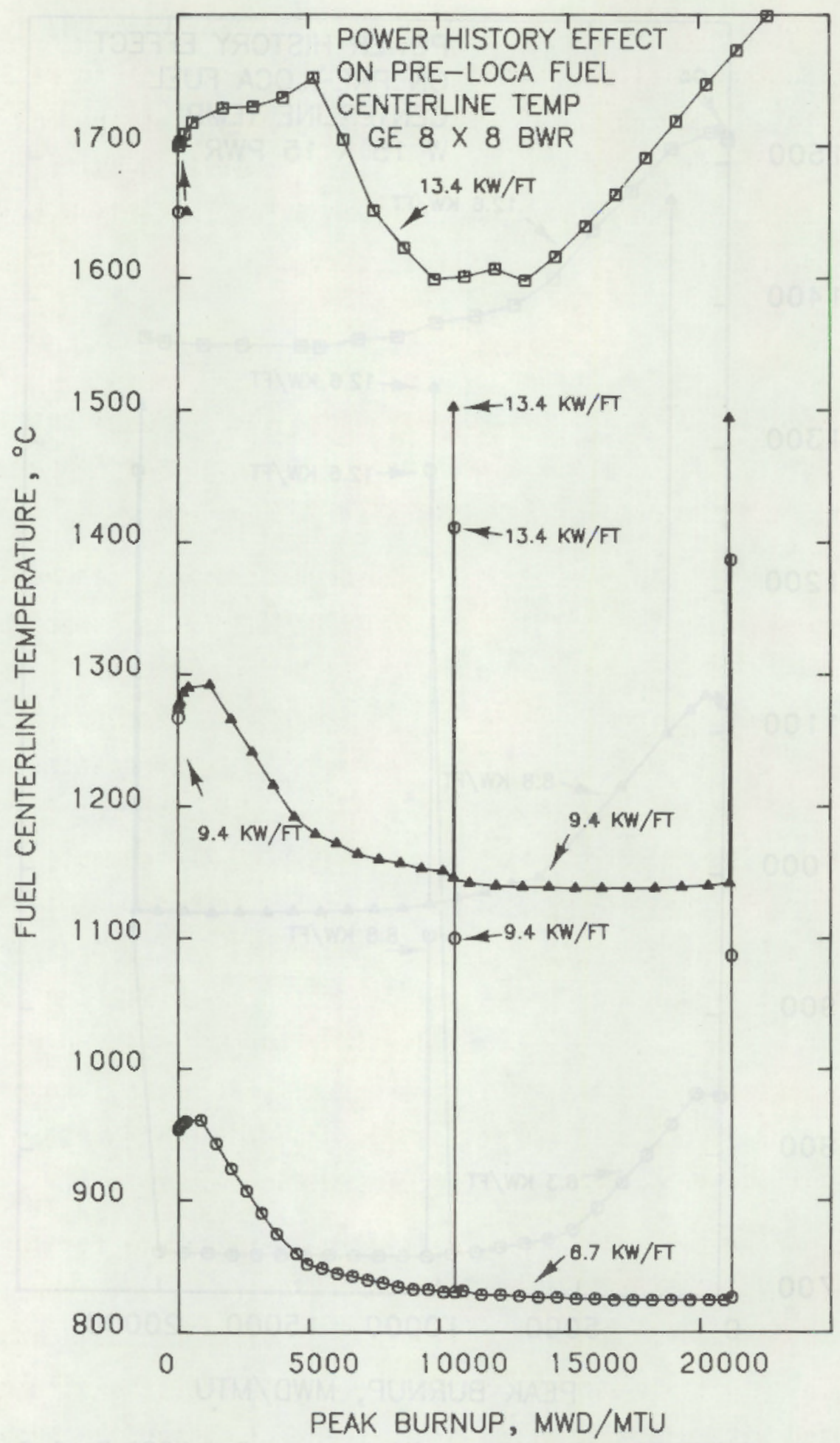

FIGURE 2. Calculated Effect of Power History on Pre-LOCA Fuel Centerline Temperature for a $8 \times 8$ BWR Rod with a Constant Peak-toAverage Power Ratio of 1.3 
The results of power ramping while maintaining a constant rod average power and altering the axial power distribution are shown in Figure 3. At $10,000 \mathrm{MWd} / \mathrm{MTU}$, the predicted fuel centerline temperature at the end of the ramp was low by $50^{\circ} \mathrm{C}$ with respect to the constant power $12.6-\mathrm{kW} / \mathrm{ft}$ curve. After the ramp at 20,000 MWd/MTU burnup, the predicted centerline temperature was $40^{\circ} \mathrm{C}$ below the constant power $12.6-\mathrm{kW} / \mathrm{ft}$ curve.

\section{DISCUSSION}

This discussion is directed toward developing the physical bases for the predicted effects of power history on the pre-LOCA fuel centerline temperature in Figures 1 to 3 and the predicted trends with burnup.

For the $15 \times 15$ PWR rod, the decrease in predicted fuel centerline temperatures that predominantly occurs prior to 7500 MWd/MTU is associated with cladding creepdown. This is characterized by the third from the last column of the appendix tables titled "CLAD RADIUS (\%) PERM." A close correspondence between the trends of the numbers in this column with the predicted decreasing centerline temperature is apparent in Tables A.1, A.3 through A.5, A.9 through A.12, and A.16 through A.18. The inability of the computed fuel centerline temperatures after a power ramp to reach the peak temperatures of the 12.6-kW/ft curve shown in Figure 1 and 3 is attributed to the greater extent of creepdown that occurs in the larger hot gap associated with the lower power cases, e.g. $-0.4 \%$ versus $-0.9 \%$ creep in Case 1 versus Cases 4 and 5 . The creepdown increases the thermal conductance due to smaller fuel-to-cladding radial gaps. Beyond 7500 MWd/MTU, fuel swelling and cladding accomnodation establish stability in the predicted fuel centerline temperature.

The initial increase in predicted centerline temperature for the BWR cases shown in Figure 2 is associated with fuel densification determined by inspection of the columns of data in Tables A.2, A.6 through A.8, and A.13 through A.15. This initial increase is followed by a significant decrease that is associated with cladding creepdown. The fuel centerline temperatures stabilize for peak burnup values above 10,000 MWd/MTU in the low-power cases 


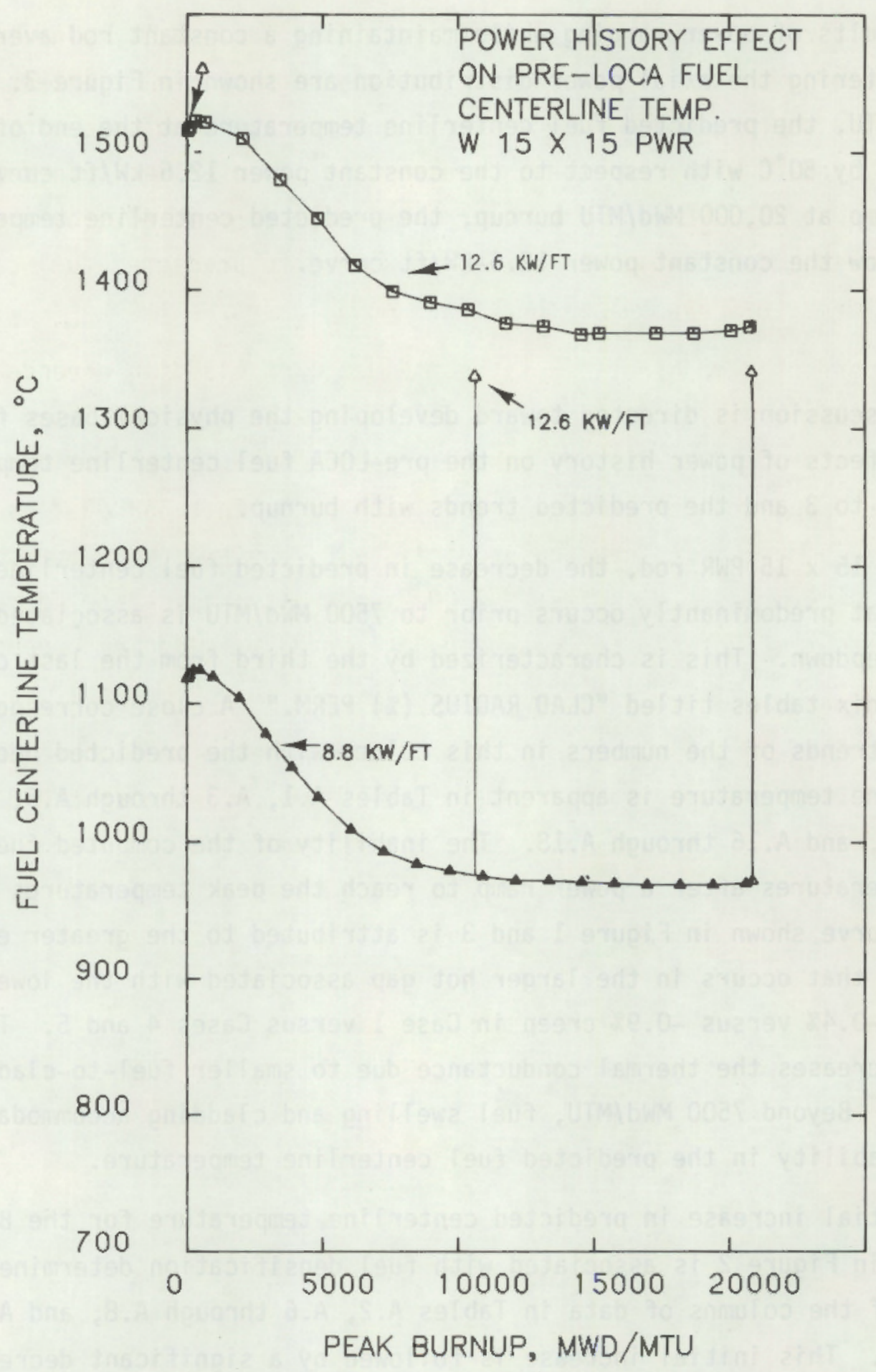

FIGURE 3. Calculated Effect of Power History on Pre-LOCA Fuel Centerline Temperature for a $15 \times 15$ PWR Rod with Constant Average Rod Power 
but not for the constant power 13.4-kW/ft case (see Table A.2). The temperatures associated with this case are sufficiently high to permit extensive release of fission gas, which reduces the gas conductivity of the gap and results in a significant predicted increase in fuel centerline temperature beyond 10,000 MWd/MTU. Except for power ramps occurring at BOL (before the onset of cladding creepdown), all power ramps lead to predicted fuel centerline temperatures that are below the higher power cases.

An independent demonstration of the influence of cladding creepdown is displayed in Figure 4. The data used for FRAPCON-2 in Tables A.1 and A.12 were also used for GAPCON-2 as described in Tables A.19 and A.20. One of the primary differences between FRAPCON-2 and GAPCON-2 is that GAPCON-2 is a creepdown-deficient code. When the predictions of the two codes are compared (see Figure 4), it can be seen that GAPCON-2 does not predict the decreasing fuel centerline temperature that has been attributed to cladding creepdown.

The level of creepdown predicted for 0.5 and 0.7 times the LOCA-1imited peak power level is on the order of $0.9 \%$ and stabilizes after a burnup of approximately $8000 \mathrm{MWd} / \mathrm{MTU}$. This level of creepdown agrees with the $0.9 \%$ creepdown reported for light water reactor (LWR) fuel rods for burnups up to 40,000 MWd/MTU. (5) 


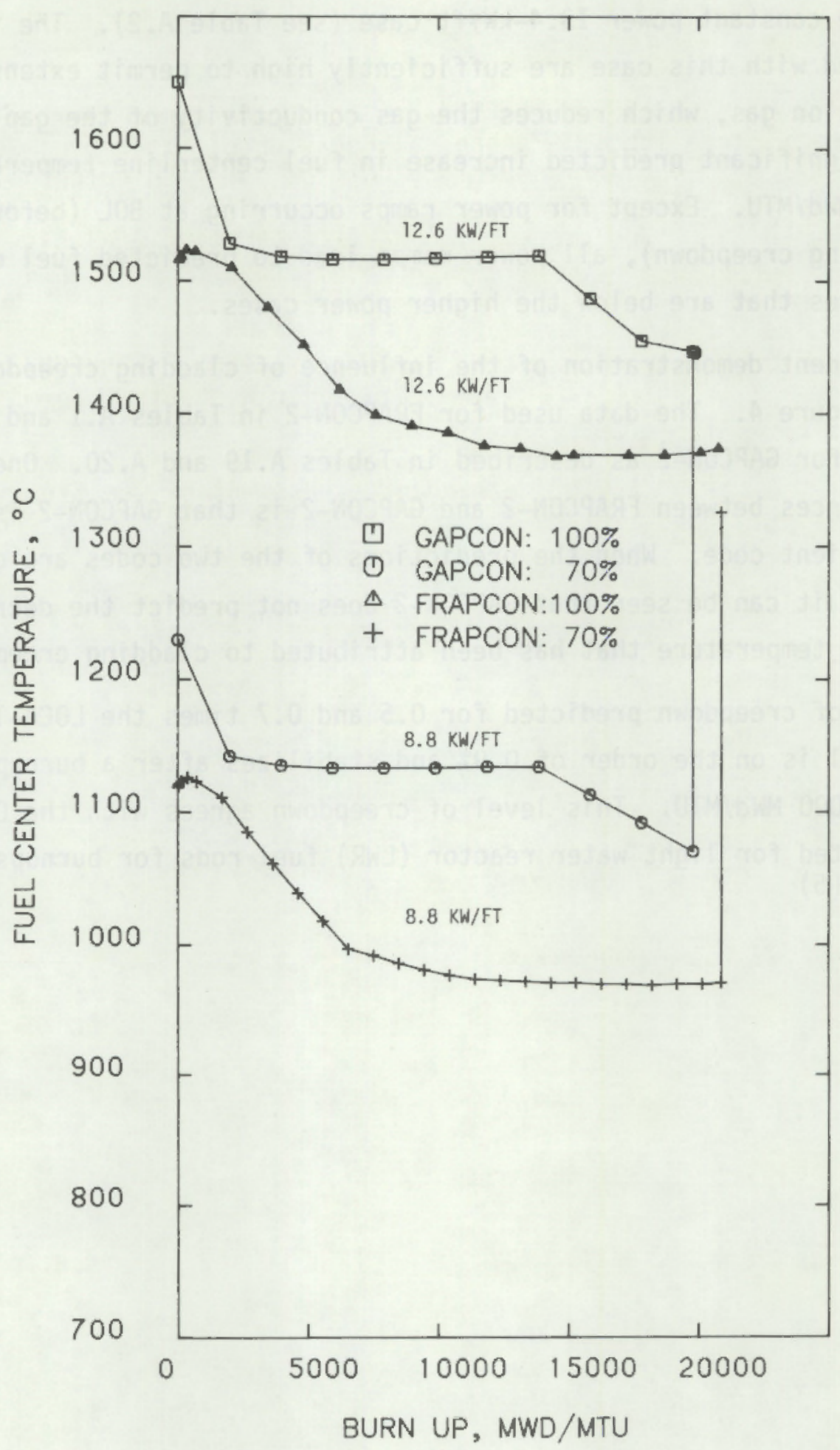

FIGURE 4. Effect of Creepdown in the FRAPCON-2 Code When Compared with the Creepdown-Deficient GAPCON-2 Code for a $15 \times 15$ PWR 


\section{$\underline{\text { REFERENCES }}$}

1. Berna, G. A., et a1. 1981. FRAPCON-2: A Computer Code for the Calculation of Steady State Thermal-Mechanical Behavior of Oxide Fuel Rods. NUREG/CR-1845, U.S. Nuclear Regulatory Commission, Washington, D.C. *

2. Acey, D. L. and J. C. Voglewede. 1980. A Comparative Analys is of LWR Fuel Designs. NUREG-0559, U.S. Nuclear Regulatory Commission, Washington, D.C. *

3. Berna, G. A., D. D. Lanning, and W. N. Rausch. 1981. FRAPCON-2 Developmental Assessment. NUREG/CR-1949, U.S. Nuclear Regulatory Commission, Washington, D.C. *

4. Laats, E. T., et al. 1981. Independent Assessment of the Steady-State Fuel Rod Analys is Code FRAPCON-2. EGG-CAAP-5335, EG\&G Idaho, Inc., Idaho Falls, Idaho.

5. Mayer, J. T., et a]. 1981. "B\&W Fuel Performance to 40,000 MWd/MTU Burnup." ANS Transactions 38:286.

*Available for purchase from the NRC/GPO Sales Program, U.S. Nuclear Regulatory Commission, Washington, DC 20555, and/or the National Technical Information Service, Springfield, VA 22161. 

APPENDIX A

SUMMARY OF COMPUTER CODE OUTPUT 
TABLE A.1. FRAPCON-2 Prediction for $15 \times 15 \mathrm{PWR}$ with $12.6 \mathrm{~kW} / \mathrm{ft}$ to $20 \mathrm{GWd} / \mathrm{MTM}, P / \mathrm{A}=1.2$

TUEL AVE AVE RADIAL FRACTION OF CUMHULATIVE ROD

\begin{tabular}{|c|c|c|c|c|c|c|c|c|c|c|c|c|c|c|c|}
\hline 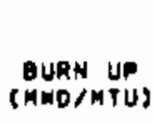 & & 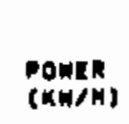 & $\begin{array}{l}\text { TUEL } \\
\text { ECNTER } \\
\text { TEMP } \\
\text { (C) }\end{array}$ & $\begin{array}{l}\text { Ave } \\
\text { puet } \\
\text { TEHP } \\
\text { (e) }\end{array}$ & $\begin{array}{l}\text { AVE } \\
\text { atoked } \\
\text { ENengY } \\
\text { (J/KO) }\end{array}$ & $\begin{array}{l}\text { RADIAL } \\
\text { OAP } \\
\text { WROTH } \\
\text { (EN) }\end{array}$ & $\begin{array}{l}\text { FRACTION OF } \\
\text { CONDUCTAMCE } \\
\text { OAP } \\
\text { CONTACT GAS }\end{array}$ & $\begin{array}{c}\text { CUMHULATIVE } \\
\text { ROD } \\
\text { FIOQ OAO } \\
\text { (X) }\end{array}$ & $\begin{array}{l}\text { ROD } \\
\text { INTERNAL } \\
\text { OAOPAES } \\
\text { (HPA) }\end{array}$ & & & $\begin{array}{l}\text { FUEL } \\
\text { JWELL } \\
(x)\end{array}$ & $\begin{array}{l}\text { CLAD } \\
\text { PERM }\end{array}$ & & 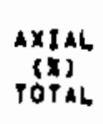 \\
\hline $\begin{array}{r}36 \\
113 \\
321 \\
667 \\
2054 \\
3440 \\
4026 \\
6213 \\
1599 \\
6966 \\
10372 \\
11759 \\
13145 \\
14531 \\
13198 \\
17304 \\
10691 \\
20077 \\
20770\end{array}$ & $\begin{array}{r}0,90 \\
2,00 \\
5,00 \\
10,00 \\
30,00 \\
50,00 \\
70,00 \\
90,00 \\
110,00 \\
130,00 \\
190,00 \\
170,00 \\
190,00 \\
3100.00 \\
230000 \\
230.00 \\
270,000 \\
390,00 \\
300,00\end{array}$ & $\begin{array}{l}41.06 \\
41.06 \\
91.06 \\
41.06 \\
41.06 \\
41.06 \\
41.06 \\
41.06 \\
41.06 \\
41.06 \\
81.06 \\
41.06 \\
41.06 \\
41.06 \\
41.06 \\
41.06 \\
41.06 \\
41.06 \\
41.06\end{array}$ & $\begin{array}{l}1516 \\
1518 \\
1523 \\
1312 \\
1510 \\
1400 \\
1452 \\
1418 \\
1399 \\
1391 \\
1306 \\
1376 \\
1374 \\
1368 \\
1369 \\
1369 \\
1349 \\
1371 \\
1374\end{array}$ & $\begin{array}{l}906 \\
908 \\
910 \\
910 \\
906 \\
893 \\
881 \\
864 \\
853 \\
849 \\
849 \\
840 \\
846 \\
644 \\
646 \\
846 \\
849 \\
651 \\
634\end{array}$ & 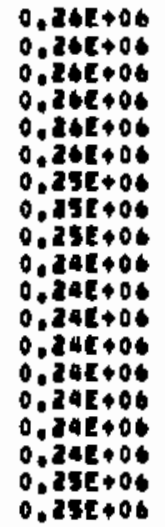 & 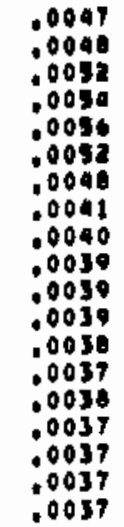 & 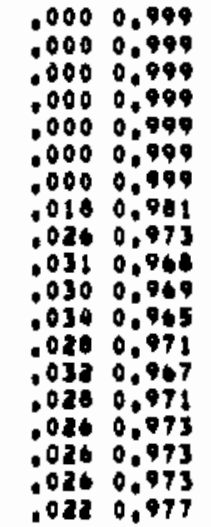 & 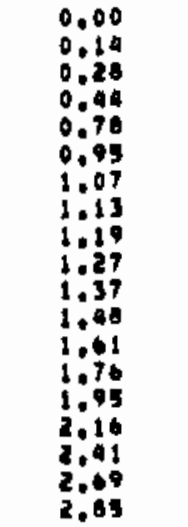 & 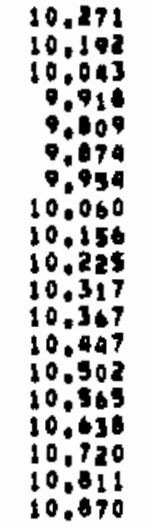 & $\begin{array}{l}.000 \\
0000 \\
0000 \\
0000 \\
0001 \\
002 \\
0002 \\
0003 \\
000 \\
0005 \\
0007 \\
.008 \\
010 \\
0012 \\
.010 \\
.017 \\
.028 \\
.025 \\
.027\end{array}$ & $\begin{array}{l}279 \\
279 \\
279 \\
279 \\
279 \\
279 \\
279\end{array}$ & 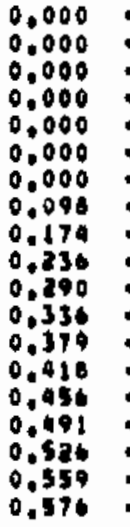 & $\begin{array}{l}96 \\
13 \\
31 \\
613 \\
13 \\
61 \\
66 \\
11 \\
97 \\
91 \\
612 \\
67 \\
20 \\
63 \\
49 \\
17 \\
85 \\
70\end{array}$ & & $\begin{array}{l}13 \\
113 \\
14 \\
14 \\
15 \\
14 \\
20 \\
20 \\
30 \\
35 \\
35 \\
39 \\
43 \\
47 \\
51 \\
55 \\
58 \\
960\end{array}$ \\
\hline
\end{tabular}


TABLE A.2. FRAPCON-2 Prediction for $8 \times 8 \mathrm{BWR}$ with $13.4 \mathrm{~kW} / \mathrm{ft}$ to $20 \mathrm{GWd} / \mathrm{MTM}, P / \mathrm{A}=1.3$

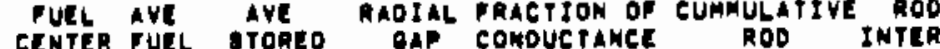

\begin{tabular}{|c|c|c|c|c|c|c|c|c|c|c|c|c|c|c|c|c|}
\hline 4 & $\begin{array}{c}\text { TIHE } \\
\text { (DAYS) }\end{array}$ & & $\begin{array}{l}\text { ENTER } \\
\text { TEMP } \\
\text { (C) }\end{array}$ & $\begin{array}{l}\text { RUE } \\
\text { Tenp } \\
\text { (C) }\end{array}$ & $\begin{array}{l}\text { MTOREO } \\
\text { CNEROY } \\
\text { (J/KO) }\end{array}$ & $\begin{array}{l}\text { OAP } \\
\text { WIOTK } \\
\text { (CN) }\end{array}$ & NOU & & riog & im & & $\begin{array}{l}\text { PUEL } \\
\text { oENA } \\
(x)\end{array}$ & (x) & $\mathrm{Cl}$ & & (3) \\
\hline 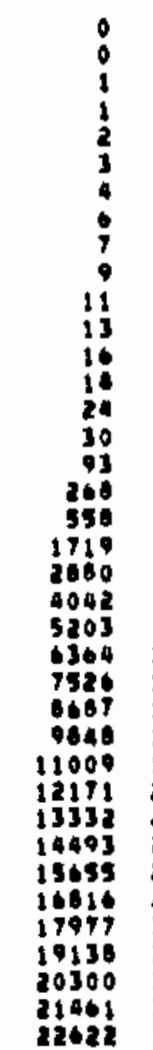 & 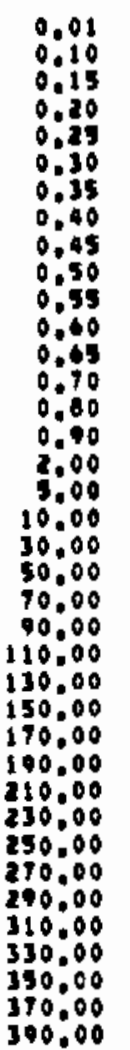 & 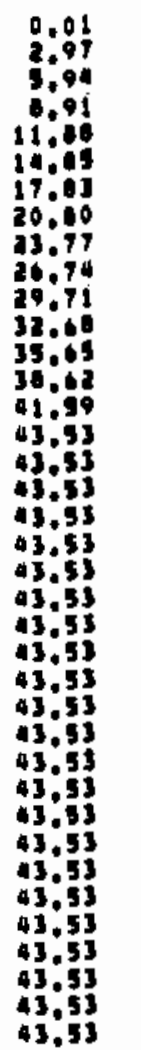 & $\begin{array}{l}278 \\
355 \\
417 \\
524 \\
117 \\
713 \\
614 \\
918 \\
1026 \\
1134 \\
1245 \\
1326 \\
1419 \\
1912 \\
1617 \\
1700 \\
1703 \\
1709 \\
1716 \\
1729 \\
1730 \\
1737 \\
1752 \\
1705 \\
1651 \\
1673 \\
1599 \\
1601 \\
1607 \\
1599 \\
1616 \\
1639 \\
1663 \\
1691 \\
1719 \\
1797 \\
1773 \\
1799\end{array}$ & 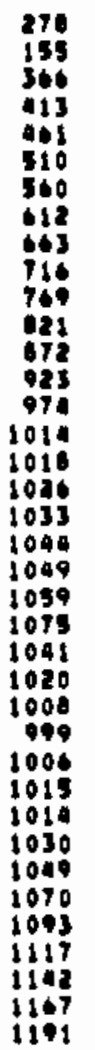 & 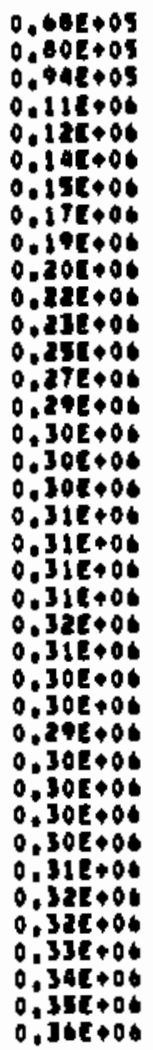 & $\begin{array}{l}0100 \\
00105 \\
00102 \\
00090 \\
00095 \\
00091 \\
00088 \\
00084 \\
0079 \\
00075 \\
00070 \\
00066 \\
00062 \\
00050 \\
00056 \\
00057 \\
00057 \\
00054 \\
00059 \\
00061 \\
00056 \\
00057 \\
00057 \\
00055 \\
00096 \\
00041 \\
00036 \\
00033 \\
00030 \\
00028 \\
00026 \\
00025 \\
00024 \\
00023 \\
00023 \\
00022 \\
00021 \\
00021\end{array}$ & 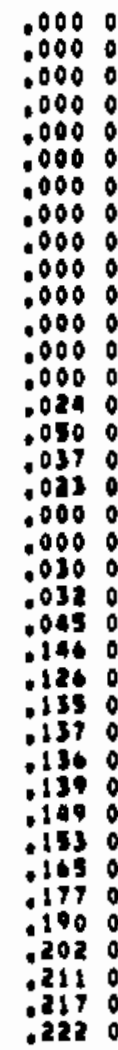 & 0,99 & 0,00 & & 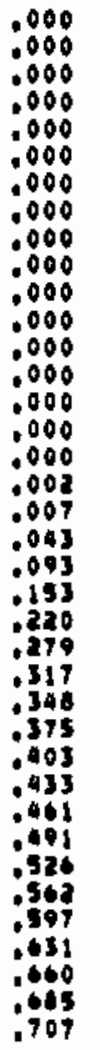 & $\begin{array}{l}200 \\
297 \\
297 \\
297 \\
297 \\
297 \\
297 \\
297 \\
297 \\
297 \\
297 \\
297 \\
297 \\
297 \\
297 \\
297 \\
297\end{array}$ & $\begin{array}{l}0.075 \\
0.923 \\
0.069 \\
1.012 \\
1.053 \\
1.092 \\
1.180 \\
1.103\end{array}$ & $\begin{array}{l}0 \\
1 \\
1 \\
1 \\
2 \\
2\end{array}$ & 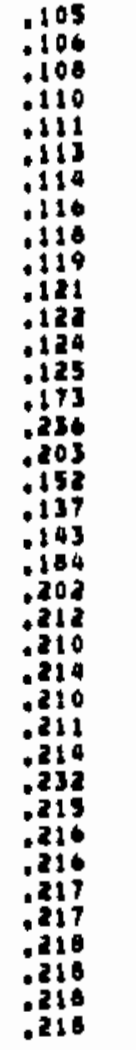 & \\
\hline
\end{tabular}


TABLE A.3. FRAPCON-2 Prediction for $15 \times 15$ PWR with $6.3 \mathrm{~kW} / \mathrm{ft}$ for 1 Day, Ramp to $12.6 \mathrm{~kW} / \mathrm{ft}, P / A=1.2$

PUEL AVE GVE RAOIAL BRACTION OF CUMMULATIVE ROD

\begin{tabular}{|c|c|c|c|c|c|c|c|c|c|c|c|c|c|c|c|}
\hline 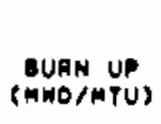 & $\begin{array}{l}\operatorname{lag} \\
\text { AYs }\end{array}$ & $\begin{array}{l}\text { POWER } \\
(K W / M)\end{array}$ & $\begin{array}{l}\text { PUEL } \\
\text { GENTER } \\
\text { TEMP } \\
\text { (C) }\end{array}$ & $\begin{array}{l}\text { AYE } \\
\text { FUEL } \\
\text { TEMF } \\
\text { (C) }\end{array}$ & $\begin{array}{l}\text { AVE } \\
\text { OTDREO } \\
\text { ENEREY } \\
\text { (JIKGS }\end{array}$ & $\begin{array}{l}\text { RAOIAL } \\
\text { GAF } \\
\text { MOOFH } \\
\text { (EM) }\end{array}$ & $\begin{array}{l}\text { ORACTION OF } \\
\text { CDNOUETANCE } \\
\text { OAP } \\
\text { CONTACT OAA }\end{array}$ & $\begin{array}{c}\text { CUMMULATIV } \\
\text { ROD } \\
\text { FIBB BAS } \\
\text { (x) }\end{array}$ & $\begin{array}{l}\text { ROD } \\
\text { INTERMAL } \\
\text { GAS PRES } \\
\text { (MPA) }\end{array}$ & $\begin{array}{l}\text { XENON } \\
\text { (NOLEE } \\
\text { PAAC) }\end{array}$ & $\begin{array}{l}\text { FLE } \\
\text { DENA } \\
\text { (x) }\end{array}$ & $\begin{array}{l}\text { PUEL } \\
\text { suELL } \\
\text { (x) }\end{array}$ & 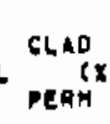 & & $\begin{array}{l}A \times I A L \\
6151 \\
107 \Delta L\end{array}$ \\
\hline $\begin{array}{l}0 \\
0 \\
0 \\
1 \\
1 \\
2 \\
3 \\
4 \\
14 \\
32 \\
33 \\
37 \\
38 \\
40 \\
42 \\
90\end{array}$ & 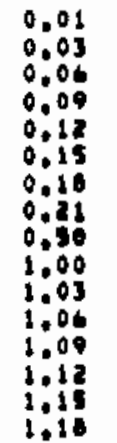 & $\begin{array}{l}0.01 \\
2.90 \\
3.90 \\
8.94 \\
11.92 \\
14.90 \\
17.90 \\
20.58 \\
20.58 \\
33.34 \\
26.32 \\
20.30 \\
32.28 \\
35.26 \\
38.24 \\
41.07\end{array}$ & $\begin{array}{l}274 \\
303 \\
616 \\
493 \\
370 \\
603 \\
758 \\
822 \\
642 \\
933 \\
1034 \\
6130 \\
1246 \\
1329 \\
9921 \\
1916\end{array}$ & $\begin{array}{l}274 \\
312 \\
352 \\
279 \\
936 \\
961 \\
526 \\
368 \\
968 \\
613 \\
601 \\
711 \\
761 \\
809 \\
950 \\
906\end{array}$ & 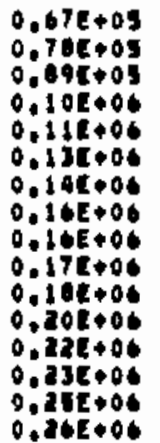 & 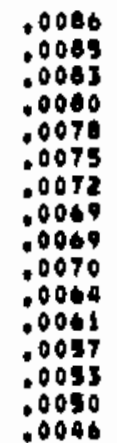 & 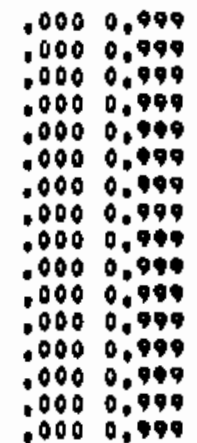 & $\begin{array}{l}0.00 \\
0.00 \\
0.00 \\
0.00 \\
0.00 \\
0.00 \\
0.00 \\
0.000 \\
0.00 \\
0.000 \\
0.00 \\
0.00 \\
0.000 \\
0.01 \\
0.02 \\
0.02\end{array}$ & 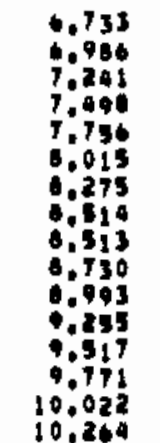 & $\begin{array}{l}.000 \\
0000 \\
0000 \\
0000 \\
0000 \\
0000 \\
0000 \\
0000 \\
0000 \\
0000 \\
0000 \\
0000 \\
0000 \\
0000 \\
.0000\end{array}$ & 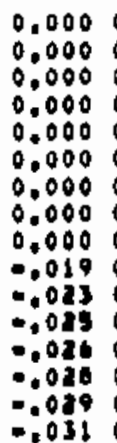 & $\begin{array}{l}0.000 \\
0.000 \\
0,000 \\
0.000 \\
0.000 \\
0.0000 \\
0.000 \\
0.000 \\
0.000 \\
0.0000 \\
0.000 \\
0.000 \\
0.0000 \\
0.000 \\
0.000 \\
0.000\end{array}$ & 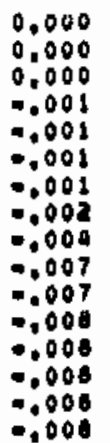 & 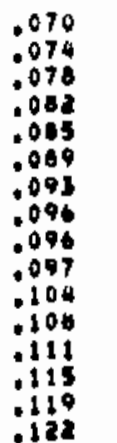 & $\begin{array}{l}0.10 \\
0: 10 \\
0: 11 \\
0.11 \\
0.11 \\
0.11 \\
0: 11 \\
0.11 \\
0.11 \\
0.11 \\
0.12 \\
0.12 \\
0.12 \\
0.12 \\
0.12 \\
0.12\end{array}$ \\
\hline
\end{tabular}


TABLE A.4. FRAPCON-2 Prediction for $15 \times 15$ PWR with $6.3 \mathrm{~kW} / \mathrm{ft}$ for $10 \mathrm{GWd} / \mathrm{MTM}$, Ramp to $12.6 \mathrm{~kW} / \mathrm{ft}, \mathrm{P} / \mathrm{A}=1.2$

\begin{tabular}{|c|c|c|c|c|c|c|c|c|c|c|c|c|c|c|c|c|}
\hline $\begin{array}{l}\text { URN UP } \\
\text { MD/MTUS }\end{array}$ & ayed & $\begin{array}{l}\text { OWER } \\
\text { KW/M) }\end{array}$ & $\begin{array}{l}\text { PUEL } \\
\text { CENTER } \\
\text { TEMP } \\
\text { (C) }\end{array}$ & $\begin{array}{l}\text { PVE } \\
\text { PUEL } \\
\text { TEFP } \\
\text { (C) }\end{array}$ & $\begin{array}{l}\text { AVE } \\
\text { STORED } \\
\text { ENERGY } \\
\text { (J/KE) }\end{array}$ & $\begin{array}{l}\text { RADIAL } \\
\text { GAP } \\
\text { WOPH } \\
\text { (EM) }\end{array}$ & $\begin{array}{l}\text { PRAC } \\
\text { CONOA } \\
\text { OA } \\
\text { CONT }\end{array}$ & & $\begin{array}{l}\text { CUMmulative } \\
\text { FtsoD } \\
(x)^{\text {nas }}\end{array}$ & $\begin{array}{c}\text { ROD } \\
\text { INIERMAL } \\
\text { EAS PARE } \\
\text { (NPA) }\end{array}$ & & 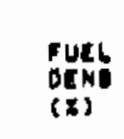 & $\begin{array}{l}\text { PUEL } \\
\text { oneth } \\
\text { (x) }\end{array}$ & & & 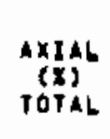 \\
\hline $\begin{array}{r}0 \\
0 \\
0 \\
1 \\
1 \\
2 \\
3 \\
4 \\
14 \\
32 \\
866 \\
1735 \\
2604 \\
3473 \\
4342 \\
5211 \\
6080 \\
6949 \\
7810 \\
8689 \\
9536 \\
10541 \\
10543 \\
10544 \\
10546 \\
10348 \\
10550 \\
10552\end{array}$ & $\begin{array}{l}0.01 \\
0.03 \\
0.06 \\
0.09 \\
0.12 \\
0 \% 13 \\
0.10 \\
0.21 \\
0.50 \\
1.00 \\
25.00 \\
50.00 \\
75.00 \\
100.00 \\
125.00 \\
150.00 \\
175,00 \\
200.00 \\
225.00 \\
250.00 \\
275.00 \\
300.00 \\
300.03 \\
300.00 \\
300.09 \\
300.12 \\
300.15 \\
300.18\end{array}$ & 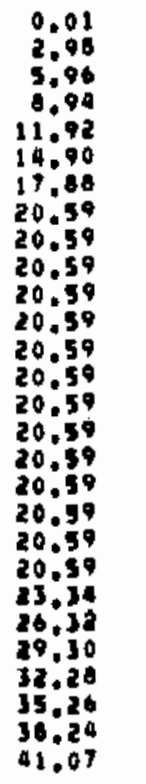 & $\begin{array}{l}279 \\
343 \\
946 \\
493 \\
376 \\
663 \\
753 \\
642 \\
842 \\
842 \\
840 \\
822 \\
800 \\
700 \\
762 \\
746 \\
736 \\
736 \\
733 \\
730 \\
728 \\
796 \\
879 \\
962 \\
1046 \\
1133 \\
1213 \\
1263\end{array}$ & $\begin{array}{l}279 \\
312 \\
392 \\
279 \\
436 \\
401 \\
526 \\
568 \\
368 \\
568 \\
370 \\
301 \\
551 \\
543 \\
536 \\
326 \\
526 \\
529 \\
532 \\
321 \\
519 \\
533 \\
594 \\
633 \\
672 \\
711 \\
748 \\
781\end{array}$ & 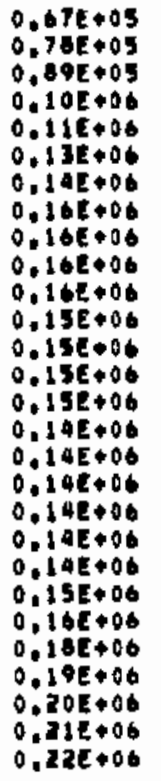 & $\begin{array}{l}.0086 \\
00003 \\
00003 \\
00000 \\
00078 \\
00075 \\
00072 \\
00069 \\
00069 \\
00070 \\
00078 \\
00072 \\
0005 \\
00058 \\
00051 \\
00044 \\
00043 \\
0002 \\
00042 \\
00042 \\
00041 \\
00042 \\
00040 \\
00041 \\
00000 \\
00000 \\
00038 \\
00035\end{array}$ & $\begin{array}{l}0000 \\
0000 \\
0000 \\
0000 \\
0000 \\
0000 \\
0000 \\
0000 \\
0000 \\
0000 \\
0000 \\
0000 \\
0000 \\
0000 \\
0000 \\
.000 \\
.020 \\
.019 \\
0016 \\
0020 \\
0023 \\
.039 \\
0034 \\
.046 \\
0054 \\
0058 \\
0061 \\
.061\end{array}$ & 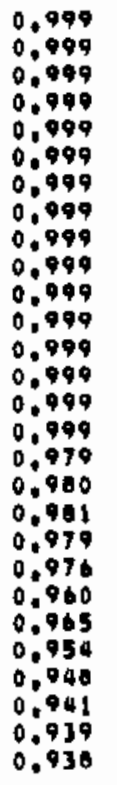 & 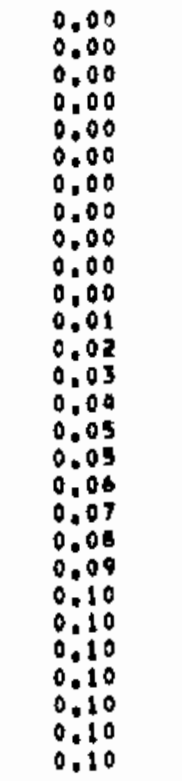 & $\begin{array}{l}6.733 \\
6.986 \\
7.241 \\
7.408 \\
7.756 \\
8.015 \\
8.275 \\
0.514 \\
8.313 \\
8.406 \\
8.216 \\
807\end{array}$ & $\begin{array}{l}0000 \\
0000 \\
0000 \\
0000 \\
0000 \\
0000 \\
0000 \\
0000 \\
0000 \\
0000 \\
0000 \\
0000 \\
0000 \\
0000 \\
0000 \\
0000 \\
0000 \\
0000 \\
0000 \\
0000 \\
0000 \\
0000 \\
0000 \\
0000 \\
0000 \\
0000 \\
0000\end{array}$ & 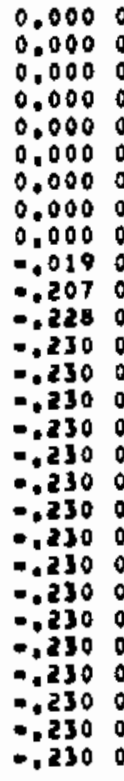 & $\begin{array}{l}0.120 \\
0.120 \\
0.170\end{array}$ & 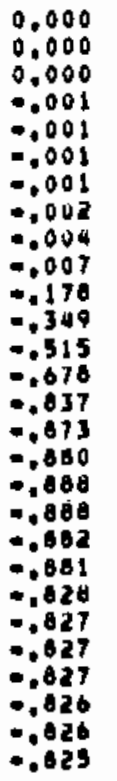 & 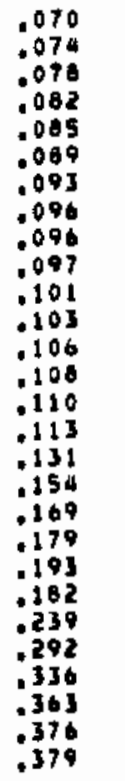 & $\begin{array}{l}0.10 \\
0.10 \\
0.11 \\
0.11 \\
0.11 \\
0.11 \\
0.11 \\
0.11 \\
0.11 \\
0.11 \\
0.13 \\
0.14 \\
0.14 \\
0.15 \\
0.15 \\
0.116 \\
0.14 \\
0.15 \\
0.16 \\
0.17 \\
0.19 \\
0.26 \\
0.31 \\
0.36 \\
0.41 \\
0.46 \\
0.50 \\
0.54\end{array}$ \\
\hline
\end{tabular}


TABLE A.5. FRAPCON-2 Prediction for $15 \times 15$ PWR with $6.3 \mathrm{~kW} / \mathrm{ft}$ for $20 \mathrm{GWd} / \mathrm{MTM}$, Ramp to $12.6 \mathrm{~kW} / \mathrm{ft}, P / A=1.2$

FUEL AVE AVE RAOIAL TRACTIOM OF CUMHULATIVE ROO

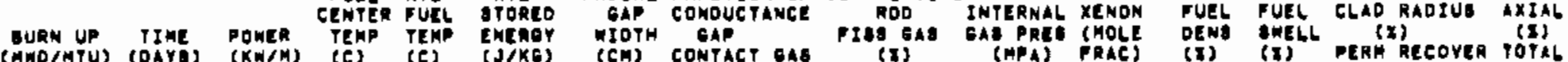

\begin{tabular}{|c|c|c|}
\hline 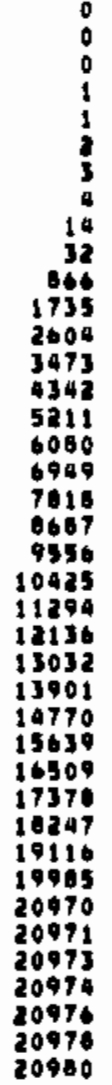 & 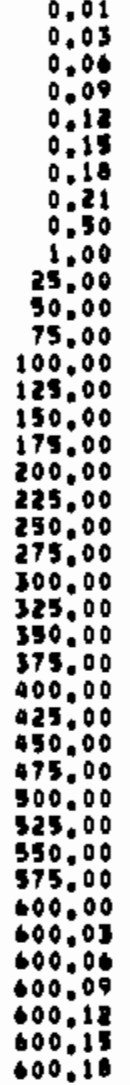 & 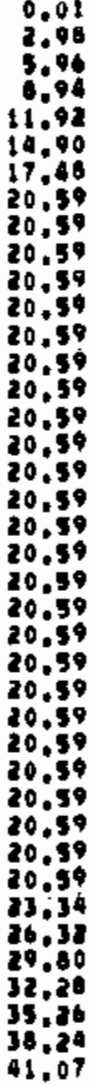 \\
\hline
\end{tabular}

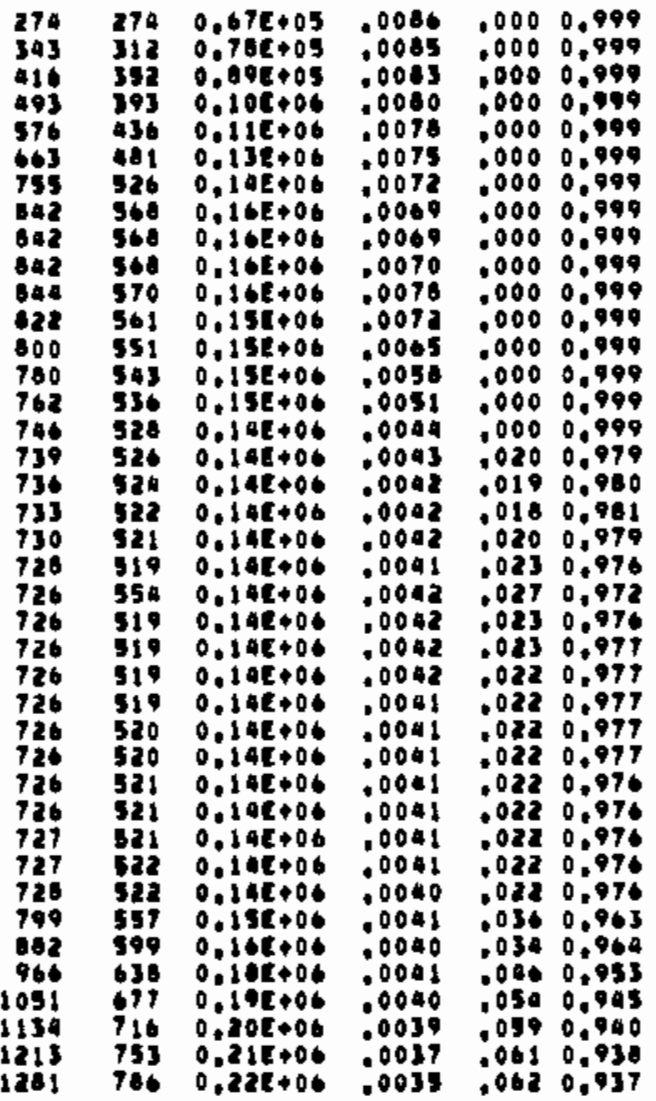

\begin{tabular}{|c|}
\hline $\begin{array}{l}100 \\
00 \\
00 \\
00 \\
00 \\
00 \\
000 \\
000 \\
00 \\
00 \\
00 \\
01 \\
002 \\
003 \\
004 \\
05 \\
05 \\
06 \\
007 \\
08 \\
09 \\
10 \\
10 \\
11 \\
112 \\
13 \\
10 \\
14 \\
15 \\
16 \\
17 \\
18 \\
19 \\
19 \\
20 \\
20 \\
20 \\
20 \\
20 \\
20 \\
20\end{array}$ \\
\hline
\end{tabular}

\begin{tabular}{|c|c|c|c|c|c|}
\hline 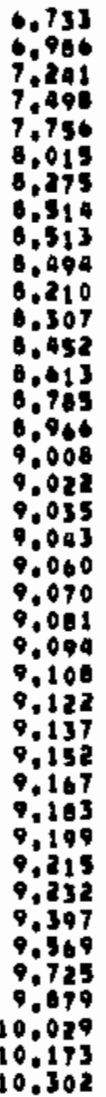 & $\begin{array}{l}000 \\
0000 \\
0000 \\
0000 \\
000 \\
000 \\
000 \\
0000 \\
0000 \\
0000 \\
0000 \\
0000 \\
0000 \\
0000 \\
000 \\
000 \\
0000 \\
0000 \\
0000 \\
0000 \\
0000 \\
0000 \\
001 \\
0001 \\
0001 \\
0001 \\
0001 \\
0001 \\
0001 \\
001 \\
0001 \\
0002 \\
0002 \\
0002 \\
0002 \\
0002 \\
0002 \\
0002 \\
0002 \\
0002\end{array}$ & 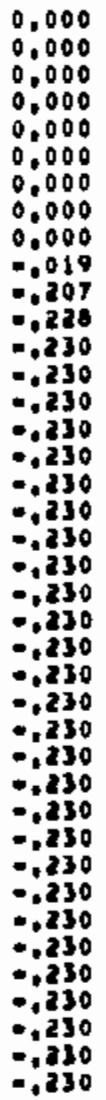 & 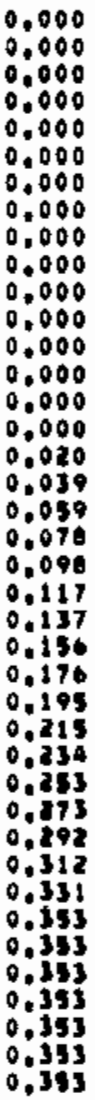 & 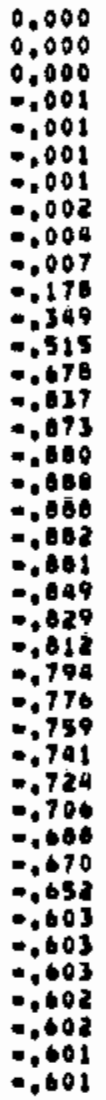 & 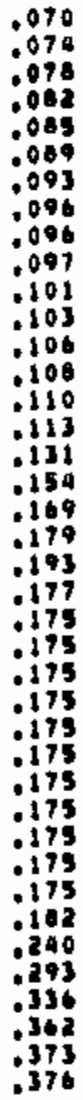 \\
\hline
\end{tabular}


TABLE A.6. FRAPCON-2 Prediction for $8 \times 8$ BWR with $6.7 \mathrm{~kW} / \mathrm{ft}$ for 1 Day, Ramp to $13.4 \mathrm{~kW} / \mathrm{ft}$, $P / A=1.3$

\begin{tabular}{|c|c|c|c|c|c|c|c|c|c|c|c|c|c|c|c|c|}
\hline $\begin{array}{l}\text { URN UP } \\
\text { WOAMfUS }\end{array}$ & $\begin{array}{l}\text { TIME } \\
\text { (OAYS) }\end{array}$ & $\begin{array}{l}\text { POWER } \\
(K W / M)\end{array}$ & $\begin{array}{l}\text { PUEL } \\
\text { CENTER } \\
\text { TEMP } \\
\text { (C) }\end{array}$ & $\begin{array}{l}\text { AVE } \\
\text { TUEL } \\
\text { TEMP } \\
\text { (C) }\end{array}$ & $\begin{array}{l}\text { AVE } \\
\text { STOAEO } \\
\text { ENEREY } \\
(J / K G)\end{array}$ & $\begin{array}{c}\text { GAOIAL } \\
\text { GAP } \\
\text { WIOTH } \\
\text { (CH) }\end{array}$ & $\begin{array}{l}\text { FRACTIC } \\
\text { CONOUE } \\
\text { GAP } \\
\text { CONTAC }\end{array}$ & $\begin{array}{l}\text { ON OF } \\
\text { TAMCE } \\
\text { S SAS }\end{array}$ & $\begin{array}{c}\text { CUMMULATIVE } \\
\text { ROD } \\
\text { Fisg GAS } \\
\text { (x) }\end{array}$ & $\begin{array}{l}\text { ROU } \\
\text { INTERNAL } \\
\text { GAB PRES } \\
\text { (HPAS) }\end{array}$ & $\begin{array}{l}\text { XENON } \\
\text { (MOLLE } \\
\text { FRAC) }\end{array}$ & $\begin{array}{l}\text { FUEL } \\
\text { DENA } \\
(x)\end{array}$ & $\begin{array}{l}\text { FUEL } \\
\text { S WELL } \\
(x) \\
\text { (x) }\end{array}$ & $\operatorname{PEHA}_{(X)}^{\text {CLAO }}$ & & $\begin{array}{c}A X I A L \\
(X) \\
r O T A L\end{array}$ \\
\hline $\begin{array}{l}0 \\
0 \\
1 \\
1 \\
2 \\
3 \\
4 \\
5 \\
73 \\
24 \\
25 \\
29 \\
20 \\
20 \\
31 \\
31 \\
34\end{array}$ & $\begin{array}{l}0.01 \\
0.10 \\
0.15 \\
0.20 \\
0.25 \\
0.30 \\
0.35 \\
0.40 \\
0.45 \\
1.00 \\
1.03 \\
1.06 \\
1.09 \\
1.12 \\
1.15 \\
1.10 \\
1.81 \\
1.24\end{array}$ & 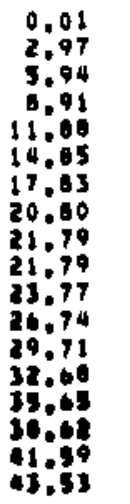 & $\begin{array}{l}278 \\
353 \\
437 \\
524 \\
617 \\
713 \\
614 \\
918 \\
954 \\
953 \\
1026 \\
1136 \\
1247 \\
1127 \\
1418 \\
2738 \\
1617 \\
1700\end{array}$ & $\begin{array}{l}278 \\
322 \\
366 \\
413 \\
401 \\
510 \\
560 \\
612 \\
629 \\
629 \\
669 \\
717 \\
769 \\
821 \\
672 \\
924 \\
974 \\
1014\end{array}$ & 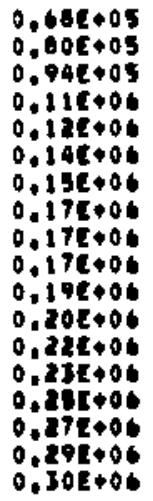 & 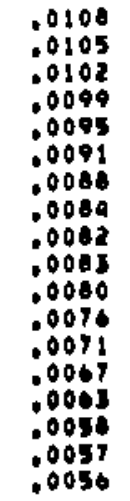 & $\begin{array}{l}.000 \\
0000 \\
0000 \\
0000 \\
0000 \\
0000 \\
0000 \\
0000 \\
0000 \\
0000 \\
0000 \\
0000 \\
0000 \\
0000 \\
0000 \\
0000 \\
0022 \\
0009\end{array}$ & $\begin{array}{l}0,999 \\
0,999 \\
0,999 \\
0,999 \\
0,999 \\
0,999 \\
0,999 \\
0,999 \\
0,999 \\
0,999 \\
0,999 \\
0,999 \\
0,999 \\
0,999 \\
0.999 \\
0.999 \\
0,979 \\
0.950\end{array}$ & $\begin{array}{l}0.00 \\
0.00 \\
0.00 \\
0.00 \\
0.00 \\
0.00 \\
0.00 \\
0.00 \\
0.00 \\
0.00 \\
0.00 \\
0.00 \\
0.00 \\
0.01 \\
0.02 \\
0.04 \\
0.00 \\
0.14\end{array}$ & $\begin{array}{l}0.216 \\
0.223 \\
0.230 \\
0.236 \\
0.243 \\
0.240 \\
0.256 \\
0.262 \\
0.264 \\
0.264 \\
0.268 \\
0.273 \\
0.279 \\
0.284 \\
0.200 \\
0.203 \\
0.290 \\
0.302\end{array}$ & $\begin{array}{l}.000 \\
.000 \\
.000 \\
1000 \\
.000 \\
.000 \\
1000 \\
.000 \\
1000 \\
.000 \\
.000 \\
.000 \\
.000 \\
.000 \\
.000 \\
.000 \\
.000 \\
.000\end{array}$ & 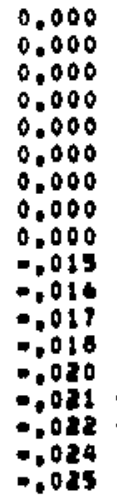 & $\begin{array}{l}0.000 \\
0.000 \\
0.000 \\
0.000 \\
0.000 \\
0.000 \\
0,000 \\
0,000 \\
0,000 \\
0,000 \\
0,000 \\
0,000 \\
0,000 \\
0.000 \\
0.000 \\
0.000 \\
0.000 \\
0,000\end{array}$ & 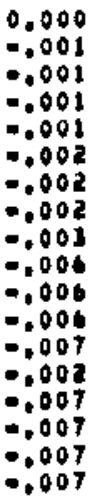 & $\begin{array}{l}.105 \\
1106 \\
1108 \\
1110 \\
1111 \\
.113 \\
1114 \\
1110 \\
1117 \\
1117 \\
1118 \\
1120 \\
1171 \\
1123 \\
1124 \\
1126 \\
1171 \\
1237\end{array}$ & $\begin{array}{l}0.11 \\
0.11 \\
0.11 \\
0.12 \\
0.12 \\
0.12 \\
0.12 \\
0.12 \\
0.12 \\
0.12 \\
0.12 \\
0.12 \\
0.12 \\
0.12 \\
0.13 \\
0.13 \\
0.12 \\
0.22\end{array}$ \\
\hline
\end{tabular}


TABLE A.7. FRAPCON-2 Prediction for $8 \times 8$ BWR with $6.7 \mathrm{~kW} / \mathrm{ft}$ for $10 \mathrm{GWd} / \mathrm{MTM}$, Ramp to $13.4 \mathrm{~kW} / \mathrm{ft}, \mathrm{P} / \mathrm{A}=1.3$

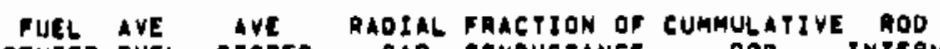

\begin{tabular}{|c|c|c|c|c|c|c|c|c|c|c|c|c|c|c|c|c|}
\hline 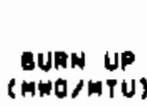 & $\begin{array}{l}\text { TIME } \\
\text { (OAYE) }\end{array}$ & $\begin{array}{l}\text { POWEA } \\
(K W / M)\end{array}$ & $\begin{array}{l}\text { CENTER } \\
\text { CENTER } \\
\text { TEWP } \\
\text { (C) }\end{array}$ & $\begin{array}{l}\text { ruet } \\
\text { TEMP } \\
\text { (C) }\end{array}$ & $\begin{array}{l}\text { aroREO } \\
\text { CNEREY } \\
\text { (J/KE) }\end{array}$ & $\begin{array}{l}\text { OAOIAL } \\
\text { OAP } \\
\text { WIDH } \\
\text { (CH) }\end{array}$ & $\begin{array}{c}\text { CONOUC } \\
\text { GAP } \\
\text { CONTACT }\end{array}$ & HCE & $\begin{array}{l}\text { ROD } \\
\text { (x) } \\
\text { GAB }\end{array}$ & $\begin{array}{l}\text { INTERNAL } \\
\text { OAB PREE } \\
\text { (MPA) }\end{array}$ & $\begin{array}{l}\text { XENON } \\
\text { (MOLLE } \\
\text { PRAC) }\end{array}$ & & $\begin{array}{l}\text { FUEL } \\
\text { aWELt } \\
\text { (x) }\end{array}$ & & & rot \\
\hline $\begin{array}{r}0 \\
\vdots \\
1 \\
2 \\
3 \\
4 \\
7 \\
23 \\
52 \\
139 \\
285 \\
866 \\
1947 \\
2020 \\
2609 \\
3191 \\
3772 \\
4533 \\
4934 \\
5515 \\
6096 \\
6678 \\
7259 \\
7040 \\
0421 \\
9002 \\
9583 \\
10165 \\
10600 \\
10601 \\
10602 \\
10604 \\
10605 \\
10606 \\
10608 \\
10610 \\
10611 \\
10669\end{array}$ & 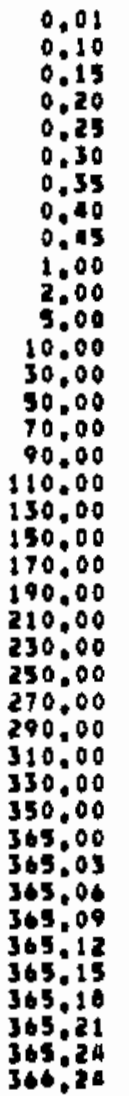 & $\begin{array}{l}0.01 \\
2.97 \\
5.94 \\
0.91 \\
11.98 \\
14.85 \\
17.83 \\
20.80 \\
21.79 \\
21.79 \\
21.79 \\
21.79 \\
21.79 \\
21.79 \\
21.79 \\
21.79 \\
21.79 \\
21.79 \\
21.79 \\
21.79 \\
21.79 \\
21.79 \\
21: 79 \\
21.79 \\
21.79 \\
21.79 \\
21.79 \\
21.79 \\
21.79 \\
21.79 \\
21.79 \\
23.77 \\
26.74 \\
29.71 \\
32.68 \\
35.65 \\
38.62 \\
41.59 \\
43.53 \\
43.53\end{array}$ & $\begin{array}{l}276 \\
353 \\
437 \\
324 \\
617 \\
913 \\
814 \\
910 \\
954 \\
955 \\
956 \\
958 \\
960 \\
961 \\
943 \\
924 \\
907 \\
490 \\
674 \\
059 \\
651 \\
648 \\
644 \\
642 \\
639 \\
637 \\
830 \\
832 \\
832 \\
630 \\
630 \\
891 \\
984 \\
1070 \\
1167 \\
1243 \\
1318 \\
1370 \\
1411 \\
1396\end{array}$ & $\begin{array}{l}278 \\
322 \\
366 \\
913 \\
461 \\
510 \\
360 \\
612 \\
629 \\
629 \\
629 \\
631 \\
631 \\
632 \\
629 \\
617 \\
609 \\
602 \\
593 \\
508 \\
506 \\
564 \\
582 \\
581 \\
579 \\
578 \\
577 \\
576 \\
577 \\
576 \\
576 \\
606 \\
649 \\
693 \\
734 \\
772 \\
770 \\
641 \\
641 \\
859\end{array}$ & 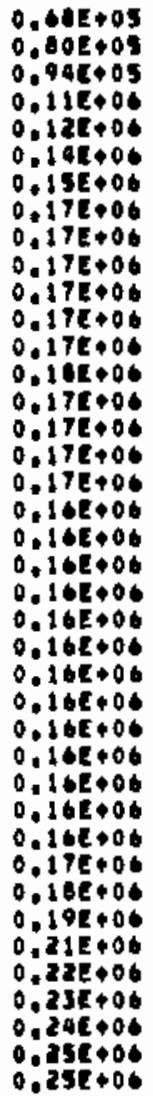 & 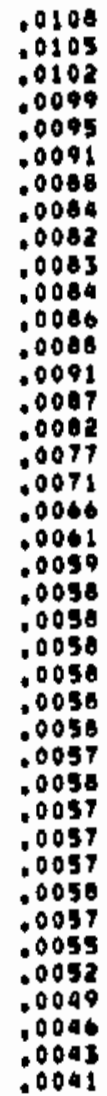 & 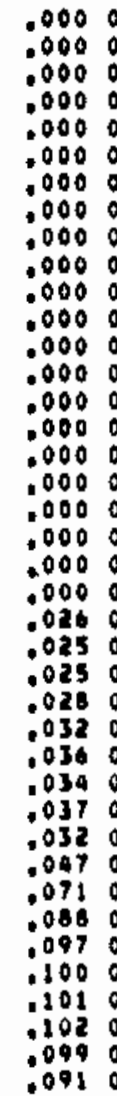 & 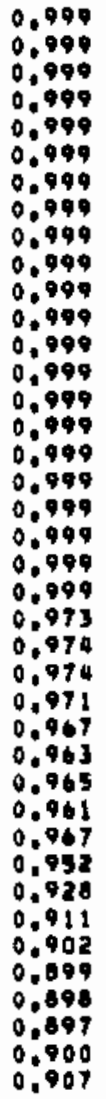 & $\begin{array}{l}0.00 \\
0.00 \\
0.00 \\
0.00 \\
0.00 \\
0.00\end{array}$ & $\begin{array}{l}73 \\
74 \\
76 \\
00 \\
63 \\
67 \\
90 \\
93 \\
95 \\
97 \\
00\end{array}$ & $\begin{array}{l}0000 \\
0000 \\
0000 \\
0000 \\
0000 \\
0000 \\
000 \\
000 \\
000 \\
0000 \\
0000 \\
000 \\
0000 \\
0000 \\
0000 \\
0000 \\
0000 \\
001 \\
0001 \\
0001 \\
0002 \\
0002 \\
0003 \\
0003 \\
0009 \\
0005 \\
0006 \\
0006 \\
0007 \\
0008 \\
0009 \\
0009 \\
0009 \\
0009 \\
0009 \\
0009 \\
0009 \\
0009 \\
0009 \\
0010\end{array}$ & 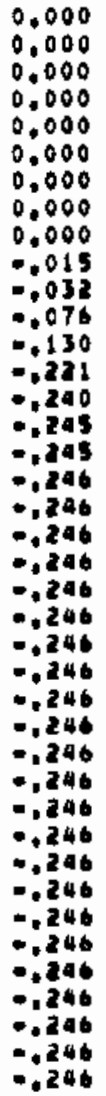 & $\begin{array}{l}0,000 \\
0.000 \\
0.000 \\
0.000 \\
0.000 \\
0.000 \\
0.000 \\
0.000 \\
0.000 \\
0.000 \\
0.000 \\
0.000 \\
0.000 \\
0.000 \\
0.000 \\
0.000 \\
0.000 \\
0.000 \\
0.000 \\
0.000 \\
0.000 \\
0.000 \\
0.013 \\
0.027 \\
0.040 \\
0.053 \\
0.067 \\
0.080 \\
0.093 \\
0.106 \\
0.116\end{array}$ & 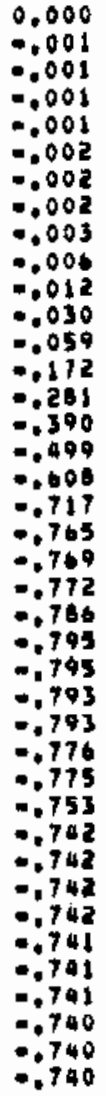 & 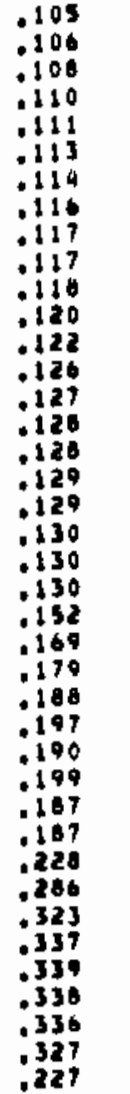 & $\begin{array}{l}11 \\
11 \\
11 \\
12 \\
12 \\
12 \\
12 \\
12 \\
12 \\
12 \\
12 \\
13 \\
13 \\
14 \\
14 \\
15 \\
15 \\
15 \\
16 \\
16 \\
16 \\
17 \\
17 \\
17 \\
16 \\
19 \\
19 \\
21 \\
21 \\
23 \\
24 \\
27 \\
32 \\
37 \\
42 \\
45 \\
49 \\
52 \\
54 \\
52\end{array}$ \\
\hline
\end{tabular}


TABLE A.8. FRAPCON-2 Prediction for $8 \times 8$ BWR with $6.7 \mathrm{~kW} / \mathrm{ft}$ for $20 \mathrm{GWd} / \mathrm{MTM}$, Ramp to $13.4 \mathrm{~kW} / \mathrm{ft}, P / A=1.3$

FUEL AYE AVE RADIAL FRACTION OF CUMMULATIVE ROU

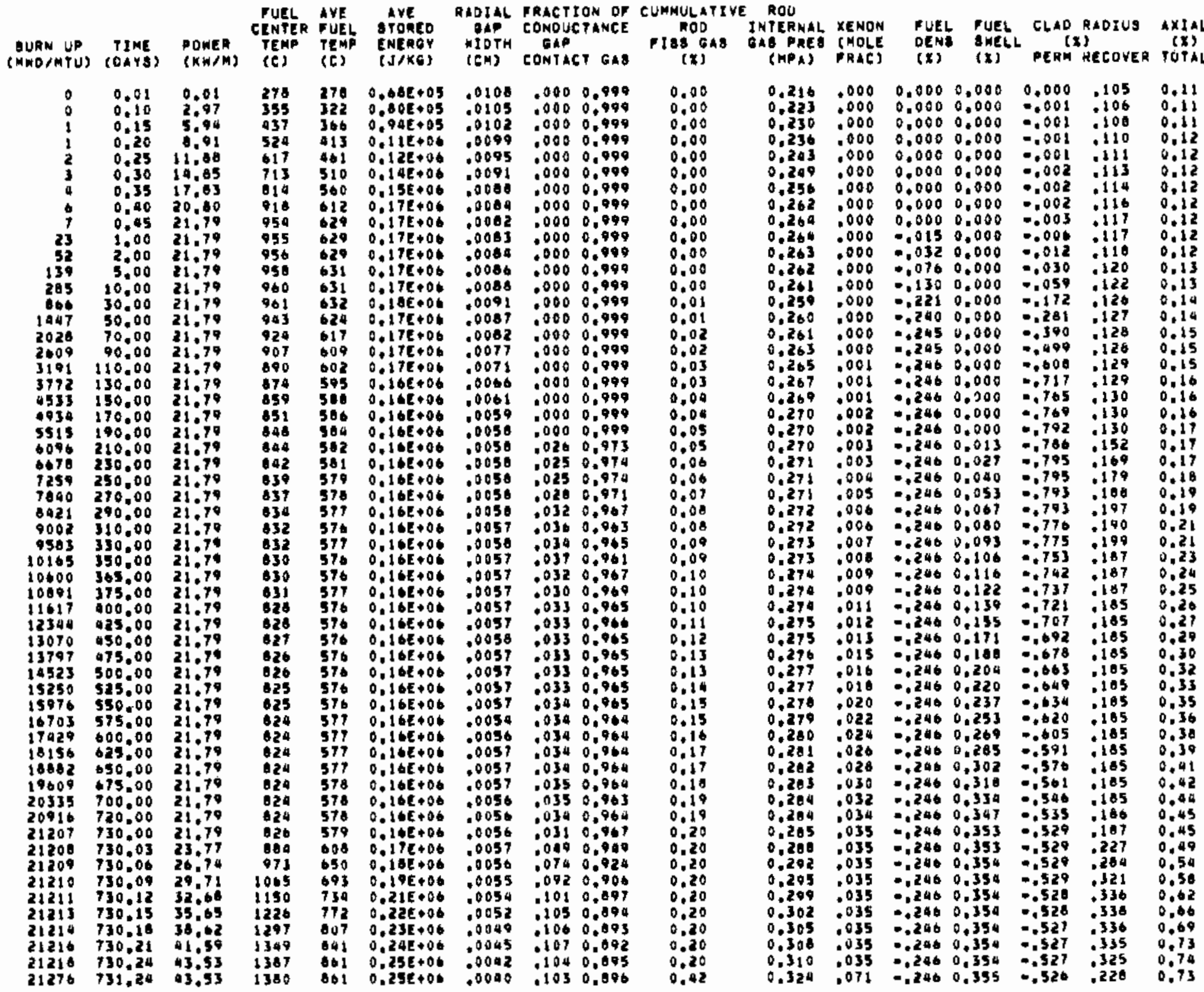


TABLE A.9. FRAPCON-2 Prediction for $15 \times 15 \mathrm{PWR}$ with $8.8 \mathrm{~kW} / \mathrm{ft}$ for 1 Day, Ramp to $12.6 \mathrm{~kW} / \mathrm{ft}$ $P / A=1.2$

\begin{tabular}{|c|c|c|c|c|c|c|c|c|c|c|c|c|c|c|c|c|}
\hline $\begin{array}{l}\text { BUAN UP } \\
\text { (HAO MTUS }\end{array}$ & $\begin{array}{c}\text { TIME } \\
\text { (Dars) }\end{array}$ & $\begin{array}{l}\text { POWER } \\
(K W / M)\end{array}$ & $\begin{array}{l}\text { FUEL } \\
\text { CENYER } \\
\text { TEMP } \\
\text { (C) }\end{array}$ & $\begin{array}{l}\text { AVE } \\
\text { TUEL } \\
\text { TEMP } \\
\text { (C) }\end{array}$ & $\begin{array}{l}\text { AVE } \\
\text { STORED } \\
\text { ENERSY } \\
\text { (J/KG) }\end{array}$ & $\begin{array}{l}\text { RAOIAL } \\
\text { GAP } \\
\text { WIOTH } \\
\text { (CA) }\end{array}$ & $\begin{array}{l}\text { SAACTIO } \\
\text { CONDUC } \\
\text { GAP } \\
\text { CONTACT }\end{array}$ & $\begin{array}{l}\text { ON OF } \\
\text { TANCE } \\
\text { TOAS }\end{array}$ & $\begin{array}{c}\text { CUMMULATIVE } \\
\text { ROD } \\
\text { FISS GAS } \\
\text { (x) }\end{array}$ & $\begin{array}{l}\text { ROU } \\
\text { INTEREAL } \\
\text { GAS PRES } \\
\text { (MPA) }\end{array}$ & $\begin{array}{l}\text { XENON } \\
\text { (MOLEE } \\
\text { FAAC) }\end{array}$ & $\begin{array}{l}\text { FUEL } \\
\text { OENA } \\
(x)\end{array}$ & $\begin{array}{l}\text { FUEL } \\
\text { GWELL } \\
(x)\end{array}$ & $\begin{array}{l}\text { CLAD } \\
=\text { PERM }\end{array}$ & RAOIUS & $\begin{array}{c}x \leq a b \\
(x) \\
T O T A L\end{array}$ \\
\hline $\begin{array}{l}0 \\
0 \\
0 \\
1 \\
1 \\
2 \\
3 \\
4 \\
5 \\
7 \\
4 \\
18 \\
42 \\
94 \\
45 \\
47 \\
49 \\
52 \\
121 \\
129 \\
132 \\
134 \\
130 \\
136 \\
140 \\
145 \\
152\end{array}$ & $\begin{array}{l}0.01 \\
0.03 \\
0.06 \\
0.00 \\
0.12 \\
0.15 \\
0.18 \\
0.21 \\
0.24 \\
0.27 \\
0.30 \\
0.30 \\
1.00 \\
1.03 \\
1.06 \\
1.09 \\
1.12 \\
1.15 \\
2.15 \\
2.30 \\
2.40 \\
2.50 \\
2.60 \\
2.70 \\
2.80 \\
2.90 \\
3.00\end{array}$ & 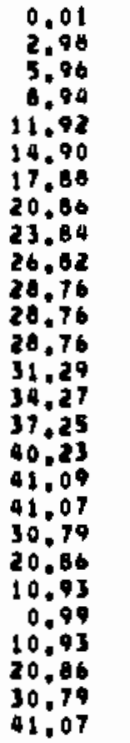 & $\begin{array}{l}274 \\
343 \\
916 \\
993 \\
576 \\
663 \\
755 \\
450 \\
999 \\
1051 \\
1119 \\
1119 \\
1121 \\
1211 \\
1317 \\
1391 \\
1908 \\
1516 \\
1513 \\
1145 \\
817 \\
532 \\
295 \\
532 \\
119 \\
1144 \\
1518\end{array}$ & $\begin{array}{l}274 \\
312 \\
352 \\
393 \\
436 \\
481 \\
526 \\
573 \\
621 \\
660 \\
701 \\
701 \\
702 \\
944 \\
794 \\
642 \\
692 \\
907 \\
900 \\
732 \\
569 \\
420 \\
287 \\
920 \\
569 \\
731 \\
907\end{array}$ & 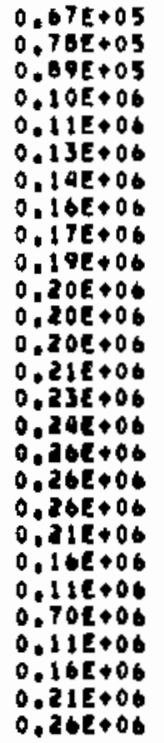 & $\begin{array}{l}.0086 \\
00085 \\
0003 \\
0000 \\
00078 \\
00075 \\
00072 \\
00070 \\
0066 \\
0063 \\
0060 \\
0000 \\
0061 \\
00058 \\
00055 \\
00051 \\
00047 \\
00046 \\
00048 \\
00062 \\
00073 \\
00082 \\
00089 \\
00083 \\
00073 \\
00061 \\
00049\end{array}$ & $\begin{array}{l}.000 \\
0000 \\
0000 \\
0000 \\
0000 \\
0000 \\
0000 \\
0000 \\
0008 \\
0008 \\
0000 \\
0000 \\
0000 \\
0000 \\
0000 \\
0000 \\
0000 \\
000 \\
0000 \\
0000 \\
0000 \\
0000 \\
0000 \\
0000 \\
0000 \\
0000 \\
0000\end{array}$ & 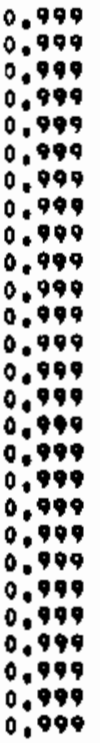 & $\begin{array}{l}0.00 \\
0.00 \\
0.00 \\
0.00 \\
0.00 \\
0.00 \\
0.00 \\
0.00 \\
0.00 \\
0.000 \\
0.000 \\
0.00 \\
0.00 \\
0.01 \\
0.01 \\
0.02 \\
0.03 \\
0.05 \\
0.16 \\
0.16 \\
0.16 \\
0.16 \\
0.16 \\
0.16 \\
0.16 \\
0.16 \\
0.17\end{array}$ & 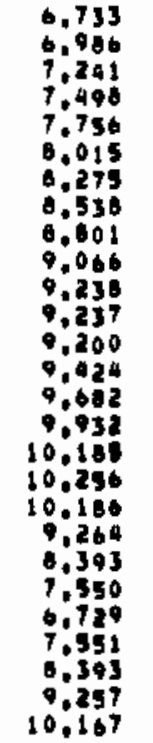 & $\begin{array}{l}.000 \\
0000 \\
0000 \\
0000 \\
0000 \\
0000 \\
000 \\
0000 \\
000 \\
0000 \\
1000 \\
0000 \\
0000 \\
0000 \\
0000 \\
0000 \\
0000 \\
0000 \\
0000 \\
0000 \\
.000 \\
0000 \\
0000 \\
0000 \\
0000 \\
0000 \\
.000\end{array}$ & 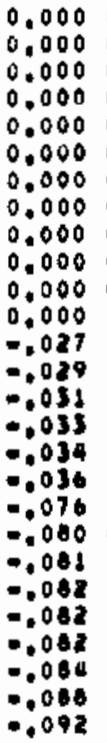 & 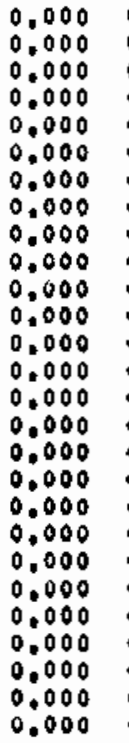 & 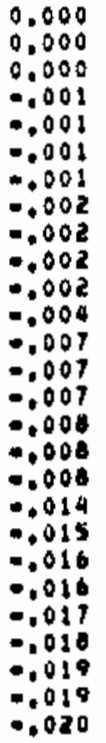 & 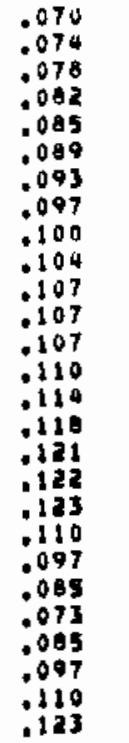 & $\begin{array}{l}0.10 \\
0.10 \\
0.11 \\
0.11 \\
0.11 \\
0.11 \\
0.11 \\
0.11 \\
0.11 \\
0.111 \\
0.12 \\
0.12 \\
0.12 \\
0.12 \\
0.12 \\
0.12 \\
0.12 \\
0.12 \\
0.12 \\
0.12 \\
0.12 \\
0.11 \\
0.11 \\
0.11 \\
0.12 \\
0.12 \\
0.12\end{array}$ \\
\hline
\end{tabular}


TABLE A.10. FRAPCON-2 Prediction for $15 \times 15$ PWR with $8.8 \mathrm{~kW} / \mathrm{ft}$ for 2 GWd/MTM, Ramp to $12.6 \mathrm{~kW} / \mathrm{ft}, P / A=1.2$

\begin{tabular}{|c|c|c|c|c|c|c|c|c|c|c|c|c|c|c|c|c|}
\hline $\begin{array}{l}\text { SURN UP } \\
A=O M T U I\end{array}$ & $\begin{array}{c}\text { TIME } \\
\text { (DAYS }\end{array}$ & $\begin{array}{l}\text { POWER } \\
(K W / M)\end{array}$ & $\begin{array}{l}\text { TUEL } \\
\text { CENTER } \\
\text { TEMP } \\
\text { (E) }\end{array}$ & $\begin{array}{l}\text { AVE } \\
\text { TUEL } \\
\text { TEMP } \\
\text { (C) }\end{array}$ & $\begin{array}{l}\text { MVE } \\
\text { OTOREO } \\
\text { CNEREY } \\
\text { (J/KS) }\end{array}$ & $\begin{array}{c}\text { AMOLAL } \\
\text { GAP } \\
\text { WIOTH } \\
\text { (CH) }\end{array}$ & $\begin{array}{c}\text { FAAETI } \\
\text { CONOUC } \\
\text { OAP } \\
\text { CONTAC }\end{array}$ & $\begin{array}{l}\text { ION OF } \\
\text { CTANEE } \\
\text { CT GAS }\end{array}$ & $\begin{array}{c}\text { CUMMULATIVE } \\
\text { ROD } \\
\text { FISs DAS } \\
\text { (E) }\end{array}$ & $\begin{array}{l}\text { ROU } \\
\text { INTERNAL } \\
\text { GAB PRES } \\
\text { (MPA) }\end{array}$ & $\begin{array}{l}\text { XENON } \\
\text { CHOLE } \\
\text { PAACS }\end{array}$ & $\begin{array}{l}\text { FUEL } \\
\text { DE ME } \\
(X)\end{array}$ & $\begin{array}{l}\text { FUEL } \\
\text { SHELL } \\
(x)\end{array}$ & $\begin{array}{c}\text { CLAD } \\
\text { PERN } \\
\text { PE }\end{array}$ & $\begin{array}{l}\text { RADIUG } \\
\text { RECOVER }\end{array}$ & $\begin{array}{l}A X I A L \\
(x) \\
\text { TOTAL }\end{array}$ \\
\hline $\begin{array}{r}0 \\
0 \\
0 \\
1 \\
1 \\
2 \\
3 \\
4 \\
5 \\
7 \\
8 \\
18 \\
42 \\
01 \\
236 \\
625 \\
1207 \\
2033 \\
2034 \\
2036 \\
2038 \\
2040 \\
2042\end{array}$ & $\begin{array}{l}0.01 \\
0.03 \\
0.06 \\
0.09 \\
0.12 \\
0.15 \\
0.110 \\
0.21 \\
0.24 \\
0.227 \\
0.30 \\
0.50 \\
1.00 \\
2.00 \\
5.00 \\
13.00 \\
25.00 \\
42.000 \\
42.03 \\
42.06 \\
42.00 \\
42.12 \\
42.15\end{array}$ & $\begin{array}{r}0.01 \\
2.98 \\
3.96 \\
8: 94 \\
11.92 \\
14.90 \\
17.88 \\
20.86 \\
23.84 \\
26.82 \\
18.76 \\
28.76 \\
28.76 \\
20.76 \\
28.76 \\
28.76 \\
28.76 \\
29.76 \\
31.29 \\
39.27 \\
37.25 \\
40.23 \\
41.07\end{array}$ & $\begin{array}{l}274 \\
343 \\
916 \\
493 \\
576 \\
663 \\
755 \\
850 \\
799 \\
1051 \\
1119 \\
1119 \\
1121 \\
1122 \\
1123 \\
1126 \\
1116 \\
1099 \\
1172 \\
1273 \\
1354 \\
1446 \\
1494\end{array}$ & $\begin{array}{l}274 \\
276 \\
352 \\
393 \\
436 \\
461 \\
526 \\
573 \\
621 \\
669 \\
701 \\
701 \\
702 \\
702 \\
703 \\
703 \\
700 \\
693 \\
728 \\
779 \\
824 \\
673 \\
807\end{array}$ & $\begin{array}{l}0.67 E+05 \\
0.76 E+05 \\
0.39 E+05 \\
0.110 E+06 \\
0.111 E+06 \\
0.13 E+06 \\
0.19 E+06 \\
0.16 E+06 \\
0.17 E+06 \\
0.19 E+06 \\
0.20 E+06 \\
0.20 E+06 \\
0.20 E+06 \\
0.20 E+06 \\
0.20 E+06 \\
0.20 E+06 \\
0.20 E+06 \\
0.19 E+06 \\
0.212 E+06 \\
0.22 E+06 \\
0.24 E+06 \\
0.25 E+06 \\
0,26 E+06\end{array}$ & 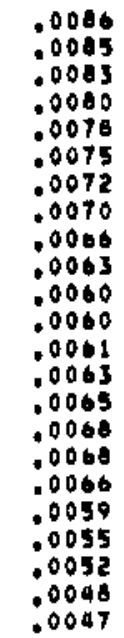 & $\begin{array}{l}.000 \\
.000 \\
0000 \\
0000 \\
0000 \\
0000 \\
0000 \\
0000 \\
000 \\
0000 \\
.000 \\
000 \\
0000 \\
0000 \\
0000 \\
0000 \\
0000 \\
0000 \\
0000 \\
0000 \\
0000 \\
.000\end{array}$ & 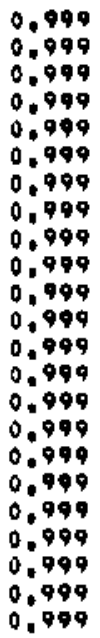 & $\begin{array}{l}0.00 \\
0.00 \\
0.00 \\
0.00 \\
0.00 \\
0.00 \\
0.00 \\
0.00 \\
0.00 \\
0.00 \\
0.00 \\
0.00 \\
0.00 \\
0.01 \\
0.01 \\
0.02 \\
0.03 \\
0.03 \\
0.03 \\
0.04 \\
0.04 \\
0.05 \\
0.05\end{array}$ & $\begin{array}{l}0.733 \\
6.986 \\
7.291 \\
7.490 \\
7.756 \\
0.015 \\
8.275 \\
6.536 \\
8.801 \\
9.066 \\
9.236 \\
9.237 \\
9.200 \\
9.162 \\
9.071 \\
8.943 \\
0.901 \\
0.925 \\
9.209 \\
9.441 \\
9.693 \\
9.902 \\
9.967\end{array}$ & $\begin{array}{l}.000 \\
.000 \\
0000 \\
0000 \\
0000 \\
0000 \\
0000 \\
0000 \\
0000 \\
0000 \\
000 \\
0000 \\
0000 \\
0000 \\
.000 \\
0000 \\
0000 \\
0000 \\
0000 \\
0000 \\
0000 \\
0000 \\
.000\end{array}$ & 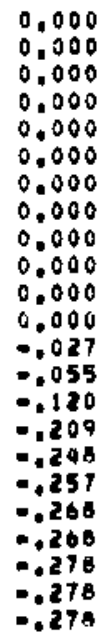 & 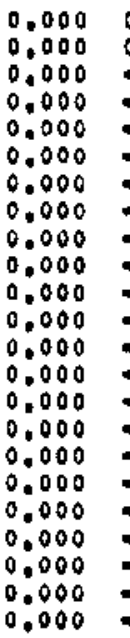 & 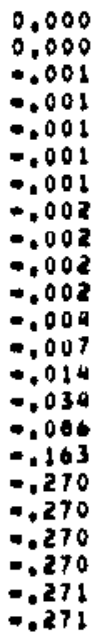 & $\begin{array}{l}.070 \\
.074 \\
.082 \\
.085 \\
.085 \\
.089 \\
.003 \\
.097 \\
1100 \\
1104 \\
1107 \\
.107 \\
1107 \\
.107 \\
1109 \\
1111 \\
1113 \\
1119 \\
1122 \\
1122 \\
1126 \\
.129 \\
.130\end{array}$ & $\begin{array}{l}0.10 \\
0.10 \\
0.11 \\
0.11 \\
0.11 \\
0.11 \\
0.11 \\
0.11 \\
0.11 \\
0.11 \\
0.12 \\
0.12 \\
0.12 \\
0.12 \\
0.12 \\
0.13 \\
0.13 \\
0.14 \\
0.14 \\
0.14 \\
0.14 \\
0.14 \\
0.14\end{array}$ \\
\hline
\end{tabular}


TABLE A.11. FRAPCON-2 Prediction for $15 \times 15$ PWR with $8.8 \mathrm{kw} / \mathrm{ft}$ for $10 \mathrm{GWd} / \mathrm{MTM}$, Ramp to $12.6 \mathrm{kH} / \mathrm{ft}, P / A=1.2$

PUEL AVE AVE RADTAL TAACTION OF CUMMULATIVE ROD

\begin{tabular}{|c|c|c|c|c|c|c|c|c|c|c|c|c|c|c|c|c|}
\hline 1 | & & & $\begin{array}{l}\text { PUEL } \\
\text { CENTER } \\
\text { TEMP } \\
\text { (C) }\end{array}$ & $\begin{array}{l}\text { AVE } \\
\text { TUEL } \\
\text { TEMP } \\
\text { (C) }\end{array}$ & $\begin{array}{l}\text { AVE } \\
\text { BTOREO } \\
\text { ENEAEY } \\
\text { (J/KG) }\end{array}$ & $\begin{array}{c}\text { RADIAL } \\
\text { GAP } \\
\text { WIOTH } \\
\text { (CH) }\end{array}$ & $\begin{array}{l}\text { ART } \\
\text { ONOU } \\
\text { SAP } \\
\text { ONA }\end{array}$ & GAB & $\begin{aligned} 280 \\
68\end{aligned}$ & $\begin{array}{l}\text { INTERNAL } \\
\text { GAS PREB } \\
\text { (MPA) }\end{array}$ & & & & & & $\begin{array}{l}A X I A L \\
(X) \\
T O T A L\end{array}$ \\
\hline $\begin{array}{r}0 \\
0 \\
1 \\
1 \\
2 \\
3 \\
4 \\
5 \\
7 \\
18 \\
42 \\
139 \\
362 \\
722 \\
1093 \\
2064 \\
3635 \\
9605 \\
5576 \\
6547 \\
7518 \\
8099 \\
9060 \\
10432 \\
10432 \\
10934 \\
10436 \\
10430 \\
10940 \\
10510 \\
10517 \\
10521 \\
10523 \\
10523 \\
10525 \\
10528 \\
10533 \\
10500\end{array}$ & 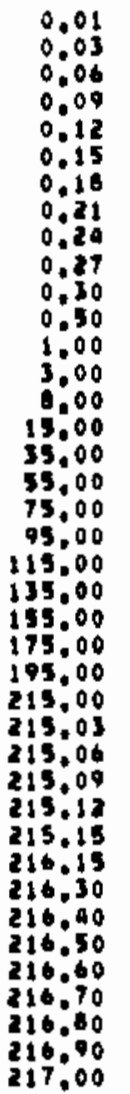 & $\begin{array}{r}0.01 \\
2.90 \\
5,90 \\
8.94 \\
11.92 \\
14.90 \\
17.91 \\
20.86 \\
23,89 \\
26.92 \\
28.76 \\
28.76 \\
28.76 \\
28.76 \\
29.76 \\
20.76 \\
29.76 \\
28.76 \\
28.76 \\
28.76 \\
28.76 \\
28.76 \\
26.76 \\
20.76 \\
28.76 \\
28.76\end{array}$ & $\begin{array}{l}214 \\
343 \\
416 \\
493 \\
576 \\
663 \\
755 \\
690 \\
949 \\
1051 \\
1119 \\
1119 \\
1121 \\
1123 \\
1126 \\
1123 \\
1111 \\
1085 \\
1061 \\
1039 \\
1018 \\
997 \\
992 \\
966 \\
981 \\
977 \\
1053 \\
1196 \\
1239 \\
1329 \\
1341 \\
1329 \\
1002 \\
730 \\
993 \\
292 \\
493 \\
730 \\
1001 \\
1303\end{array}$ & $\begin{array}{l}274 \\
312 \\
352 \\
393 \\
436 \\
481 \\
526 \\
573 \\
621 \\
669 \\
701 \\
701 \\
702 \\
703 \\
704 \\
702 \\
690 \\
680 \\
678 \\
669 \\
661 \\
652 \\
649 \\
647 \\
644 \\
642 \\
677 \\
718 \\
759 \\
799 \\
806 \\
802 \\
659 \\
523 \\
390 \\
283 \\
396 \\
523 \\
659 \\
796\end{array}$ & 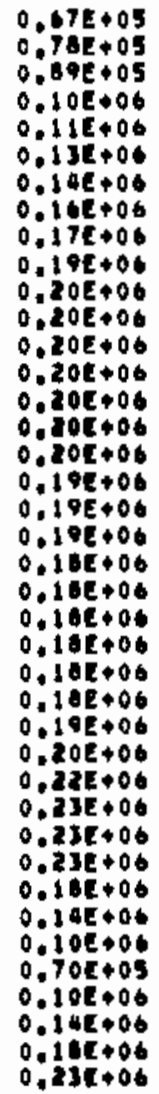 & 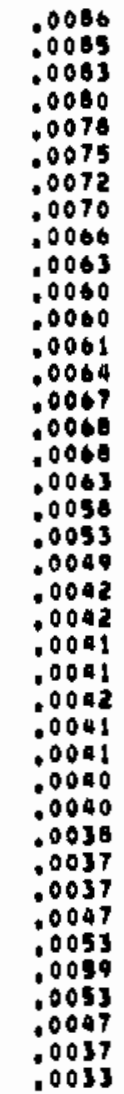 & 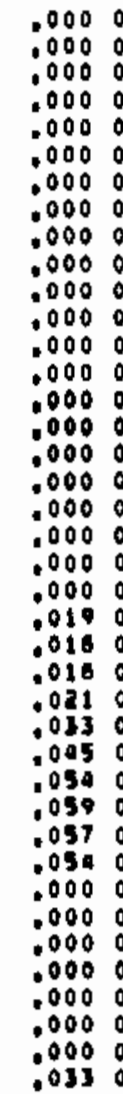 & 3 & & $\begin{array}{l}7 \\
7 \\
0 \\
8 \\
0 \\
9 \\
9 \\
9 \\
9 \\
8 \\
0\end{array}$ & $\begin{array}{l}0000 \\
0000 \\
0000 \\
0000 \\
0000 \\
0000 \\
0000 \\
0000 \\
0000 \\
0000 \\
0000 \\
0000 \\
0000 \\
0000 \\
0000 \\
0000 \\
0000 \\
0000 \\
0000 \\
0000 \\
0000 \\
0000 \\
0000 \\
0000 \\
0000 \\
0000 \\
0000 \\
0000 \\
0000 \\
0000 \\
0000 \\
0001 \\
0001 \\
0001 \\
0001 \\
0001 \\
0001 \\
0001 \\
0001 \\
0001\end{array}$ & $\begin{array}{l}0 \\
0 \\
0 \\
0 \\
0 \\
0 \\
0 \\
0 \\
0 \\
0 \\
0 \\
0 \\
0 \\
0 \\
7\end{array}$ & $\begin{array}{l}0.000 \\
0.000\end{array}$ & $\begin{array}{l}0 \\
= \\
-1\end{array}$ & 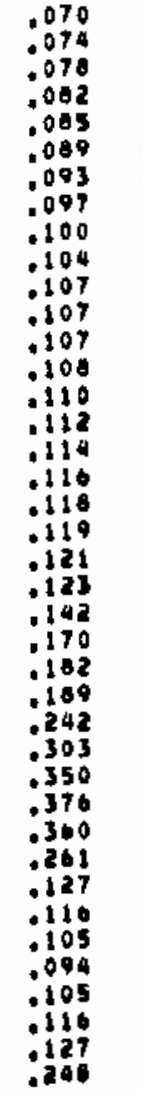 & 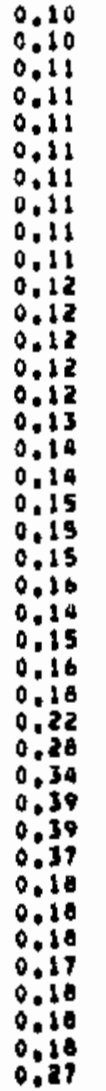 \\
\hline
\end{tabular}


TABLE A.12. FRAPCON-2 Prediction for $15 \times 15$ PWR with $8.8 \mathrm{~kW} / \mathrm{ft}$ for $20 \mathrm{GWd} / \mathrm{MTM}$, Ramp to $12.6 \mathrm{~kW} / \mathrm{ft}, P / A=1.2$

PUEL AVE AVE RADIAL BRACTION OF CUMMULATIVE ROD

\begin{tabular}{|c|c|c|c|c|c|c|c|c|c|c|c|c|c|c|c|c|}
\hline $\begin{array}{l}\text { gunN UP } \\
\text { (mMo/niv) }\end{array}$ & $\begin{array}{l}\text { TIME } \\
\text { (DAYE) }\end{array}$ & $\begin{array}{l}\text { Pomen } \\
(x \in / N)\end{array}$ & $\begin{array}{l}\text { PutL } \\
\text { eEvien } \\
\text { PEHe } \\
\text { (C) }\end{array}$ & $\begin{array}{l}\text { AVE } \\
\text { PUEL } \\
\text { TEMF } \\
\text { (C) }\end{array}$ & $\begin{array}{l}\text { Ave } \\
\text { stones } \\
\text { entensy } \\
\text { (J/ke) }\end{array}$ & $\begin{array}{c}\text { RADIAL } \\
\text { EAF } \\
\text { WIOTH } \\
\text { (CW) }\end{array}$ & $\begin{array}{l}\text { Dactio } \\
\text { CONOUCT } \\
\text { OAR } \\
\text { CONTACT }\end{array}$ & $\begin{array}{l}\text { gON OF } \\
\text { STAMEE } \\
\text { ST OAO }\end{array}$ & $\begin{array}{l}\text { QUMAULATIVE } \\
\text { ROOD } \\
\text { (E) }\end{array}$ & 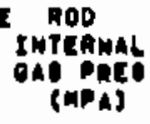 & $\begin{array}{l}\text { xewow } \\
\text { (mout } \\
\text { Prect }\end{array}$ & $\begin{array}{l}\text { FuEt } \\
\text { Dent } \\
\text { (E) }\end{array}$ & $\begin{array}{l}\text { pued } \\
\text { yutit } \\
\text { (x) }\end{array}$ & & & STAL \\
\hline 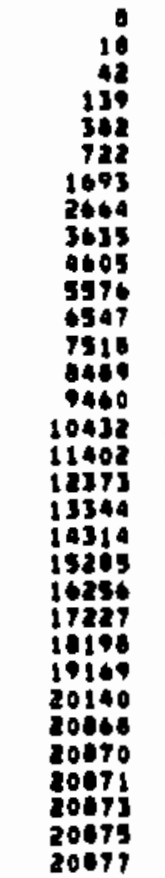 & 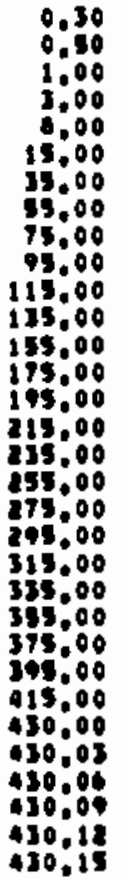 & 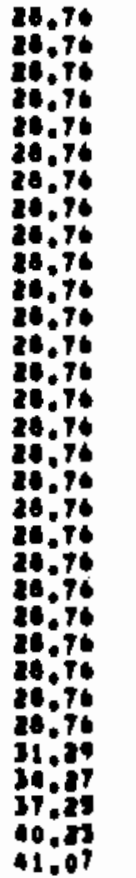 & 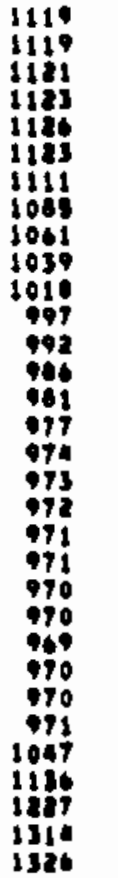 & 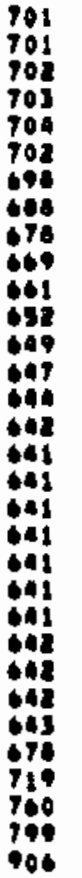 & 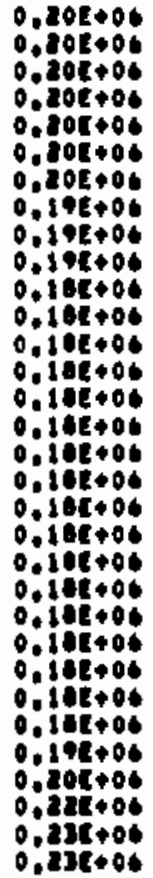 & $\begin{array}{l}00060 \\
00060 \\
00061 \\
00060 \\
00067 \\
00048 \\
00068 \\
00063 \\
00050 \\
00053 \\
00049 \\
00042 \\
00042 \\
00041 \\
00041 \\
00042 \\
00042 \\
00002 \\
00042 \\
00042 \\
00041 \\
00041 \\
00041 \\
00040 \\
08040 \\
00040 \\
00041 \\
00040 \\
00040 \\
00040 \\
00039 \\
00037\end{array}$ & 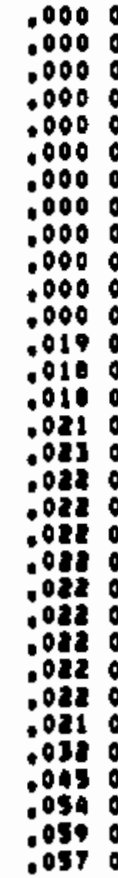 & 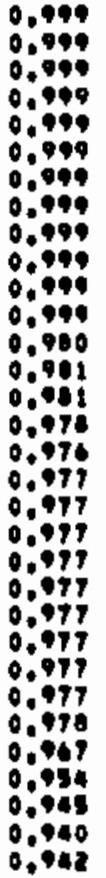 & $\begin{array}{l}0.00 \\
0.00 \\
0.00 \\
0.01 \\
0.01 \\
0.02 \\
0.03 \\
0.00 \\
0.04 \\
0.05 \\
0.09 \\
0.06\end{array}$ & 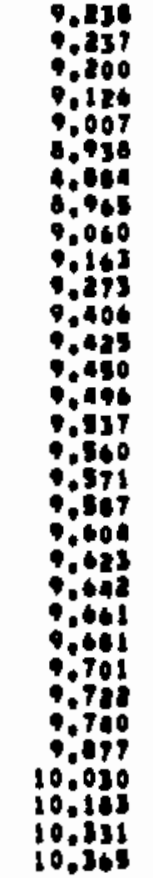 & $\begin{array}{l}0000 \\
0000 \\
0000 \\
0000 \\
0000 \\
0000 \\
0000 \\
0000 \\
0000 \\
0000 \\
0000 \\
0000 \\
0000 \\
0000 \\
0000 \\
0000 \\
0001 \\
0001 \\
0001 \\
0001 \\
0001 \\
0001 \\
0001 \\
0001 \\
0002 \\
0002 \\
0002 \\
0002 \\
0008 \\
0008 \\
0008 \\
0002\end{array}$ & 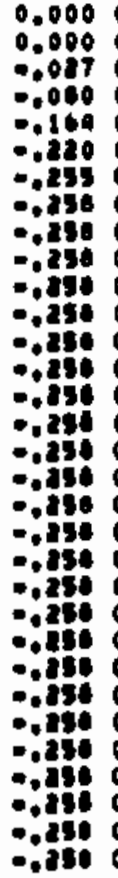 & $\begin{array}{l}0.000 \\
0.000 \\
0.000 \\
0,000 \\
0.000 \\
0,000 \\
0.000 \\
0,000 \\
0.000 \\
0,000\end{array}$ & 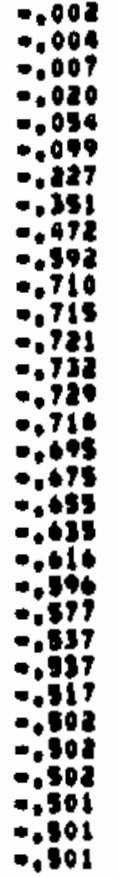 & 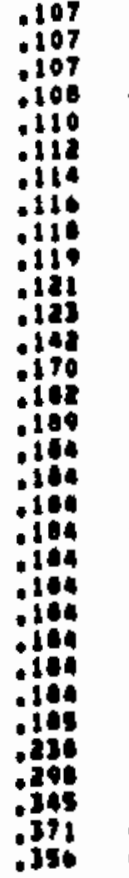 & 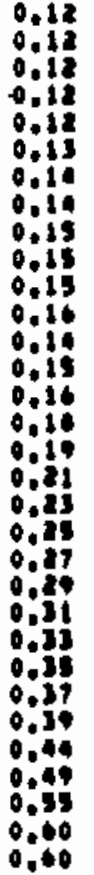 \\
\hline
\end{tabular}


TABLE A.13. FRAPCON-2 Prediction for $8 \times B$ BWR with $9.4 \mathrm{~kW} / \mathrm{ft}$ for 1 Day, Ramp to $13.4 \mathrm{~kW} / \mathrm{ft}$, $P / A=1.3$

\begin{tabular}{|c|c|c|c|c|c|c|c|c|c|c|c|c|c|c|c|c|}
\hline $\begin{array}{l}\text { IWRN UP } \\
\text { Ino/mTUS }\end{array}$ & $\begin{array}{l}\text { Tyint } \\
\text { (Dires) }\end{array}$ & $\begin{array}{l}\text { powen } \\
\text { (Ku/N) }\end{array}$ & 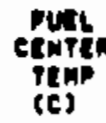 & $\begin{array}{l}\text { avi } \\
\text { putch } \\
\text { tes }\end{array}$ & 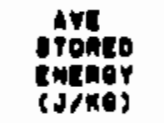 & $\begin{array}{c}\text { MADLAL } \\
\text { GAP } \\
\text { noTn } \\
\text { (en) }\end{array}$ & 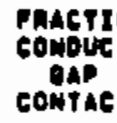 & $\begin{array}{l}\text { tow of } \\
\text { cranct } \\
\text { ct oat }\end{array}$ & 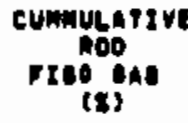 & 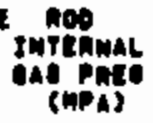 & 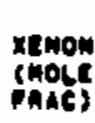 & $\begin{array}{l}\text { Futst } \\
\text { ofins } \\
\text { (x) }\end{array}$ & $\begin{array}{l}\text { purt } \\
\text { (x) }\end{array}$ & CLA & & $\begin{array}{l}\text { AxJAL } \\
\text { Tot? }\end{array}$ \\
\hline $\begin{array}{l}0 \\
0 \\
1 \\
3 \\
3 \\
\vdots \\
11 \\
13 \\
39 \\
31 \\
35 \\
37\end{array}$ & 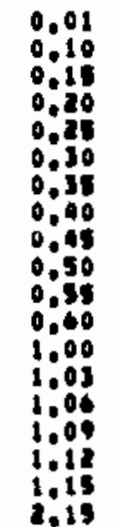 & 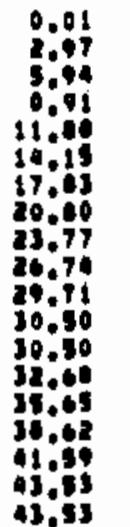 & $\begin{array}{l}278 \\
391 \\
637 \\
620 \\
617 \\
713 \\
610 \\
1036 \\
1130 \\
624 \\
1274 \\
1277 \\
1327 \\
1416 \\
1514 \\
1617 \\
1700 \\
1703\end{array}$ & $\begin{array}{l}218 \\
312 \\
360 \\
113 \\
461 \\
610 \\
960 \\
612 \\
663 \\
716 \\
761 \\
763 \\
764 \\
921 \\
078 \\
724 \\
971 \\
1019 \\
1010\end{array}$ & 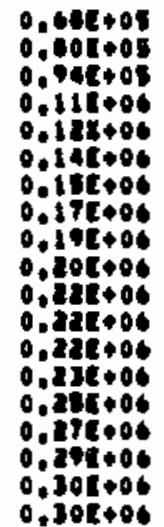 & $\begin{array}{l}.0100 \\
00105 \\
00101 \\
00101 \\
00095 \\
00001 \\
00086 \\
00084 \\
00079 \\
00075 \\
00078 \\
00069 \\
00070 \\
00067 \\
00064 \\
00058 \\
00057 \\
00057\end{array}$ & $\begin{array}{l}.000 \\
.000 \\
0000 \\
0000 \\
0000 \\
.000 \\
0000 \\
0000 \\
0000 \\
0000 \\
0000 \\
0000 \\
0000 \\
.000 \\
.000 \\
.000 \\
.020 \\
.040 \\
.037\end{array}$ & 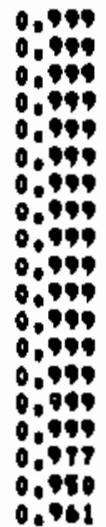 & $\begin{array}{l}0,00 \\
0,00 \\
0,00 \\
0,00 \\
0,00 \\
0,00 \\
0,00 \\
0,00 \\
0,00 \\
0,00\end{array}$ & 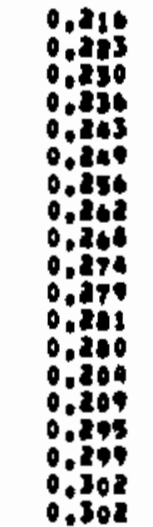 & $\begin{array}{l}.000 \\
0000 \\
0000 \\
0000 \\
0000 \\
0000 \\
0000 \\
0000 \\
0000 \\
0000 \\
0000 \\
0000 \\
0000 \\
0000 \\
.0000 \\
.000 \\
0000 \\
0000 \\
0000\end{array}$ & $\begin{array}{l}0,000 \\
0,080 \\
0,000 \\
0,000 \\
0,000 \\
0,000 \\
0,000 \\
0,000 \\
0,000 \\
0,000 \\
0,000 \\
0,000 \\
0,030 \\
0,089 \\
0,083 \\
0,085 \\
0,086 \\
0,087 \\
0,064\end{array}$ & $\begin{array}{l}0,000 \\
0,000 \\
0,000 \\
0,000 \\
0,000 \\
0,000 \\
0,000 \\
0,000 \\
0,000 \\
0,000 \\
0,000 \\
0.000\end{array}$ & 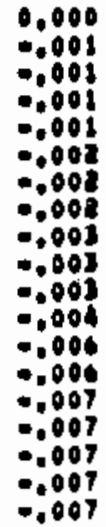 & 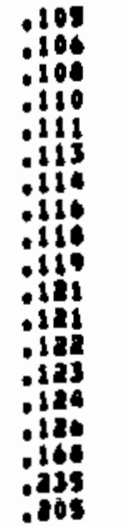 & $\begin{array}{l}0.11 \\
0.11 \\
0.11 \\
0.12 \\
0.12 \\
0.12 \\
0.12 \\
0,12 \\
0.12 \\
0.11 \\
0.12 \\
0.12 \\
0.12 \\
0.12 \\
0.13 \\
0.13 \\
0.12 \\
0.22 \\
0.20\end{array}$ \\
\hline
\end{tabular}


TABLE A.14. FRAPCON-2 Prediction for $8 \times 8 \mathrm{BWR}$ with $9.4 \mathrm{~kW} / \mathrm{ft}$ for $10 \mathrm{GWd} / \mathrm{MTM}$, Ramp to $13.4 \mathrm{~kW} / \mathrm{ft}, \mathrm{P} / \mathrm{A}=1.3$

Pute ave ave Radial maction of Cumblative noo

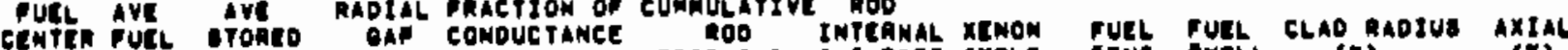

\begin{tabular}{|c|c|c|c|c|c|c|c|c|c|c|c|c|c|c|c|}
\hline $\begin{array}{l}\text { OURN UP } \\
\text { (MWO/ATUS }\end{array}$ & $\begin{array}{l}\text { TIME } \\
\text { (Divo) }\end{array}$ & $\begin{array}{l}\text { Powes } \\
(\mathrm{KH} / \mathrm{A})\end{array}$ & $\begin{array}{l}\text { sevten } \\
\text { Tent } \\
\text { (C) }\end{array}$ & $\begin{array}{l}\text { putat } \\
\text { (c) }\end{array}$ & $\begin{array}{l}\text { etonto } \\
\text { enthey } \\
\text { (J/Ke) }\end{array}$ & $\begin{array}{l}\text { naph } \\
\text { (en) }\end{array}$ & $\begin{array}{l}\text { CONDUC TANCE } \\
\text { OAP } \\
\text { CONFACT DAS }\end{array}$ & $\begin{array}{c}200 \\
\operatorname{rise} 6)^{2}\end{array}$ & $\begin{array}{l}\text { INTEANAL } \\
\text { SACPREO } \\
\text { (MPA) }\end{array}$ & TMOLE & $\begin{array}{l}\text { Fut } \\
\text { Dint } \\
\text { (5) }\end{array}$ & $\begin{array}{l}\text { FutL } \\
\text { (x)tL } \\
\text { (x) }\end{array}$ & CLA & $\begin{array}{l}\text { orve } \\
\text { coven }\end{array}$ & $\begin{array}{l}\text { AxIAL } \\
\text { (E) } \\
\text { TOTAL }\end{array}$ \\
\hline 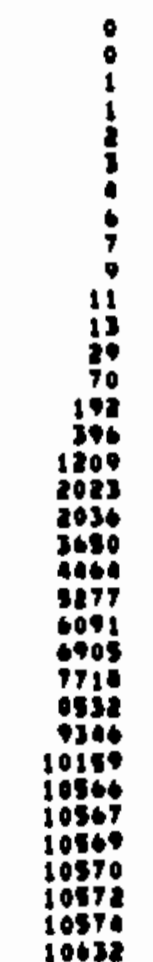 & 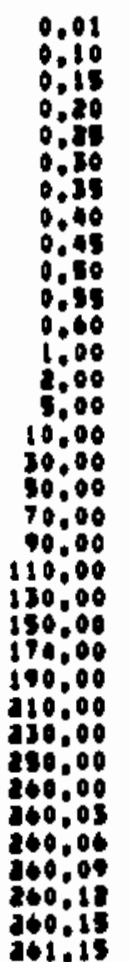 & 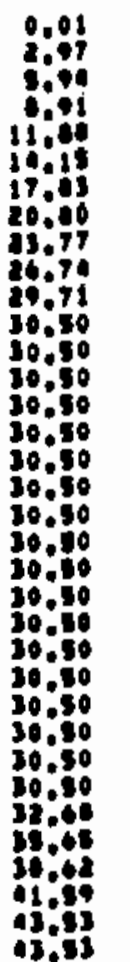 & 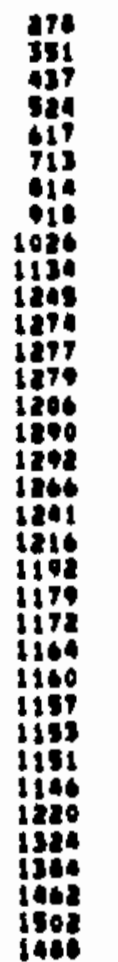 & 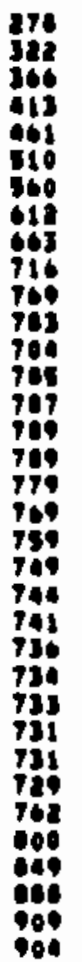 & 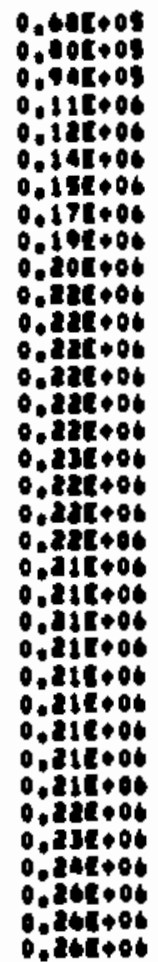 & 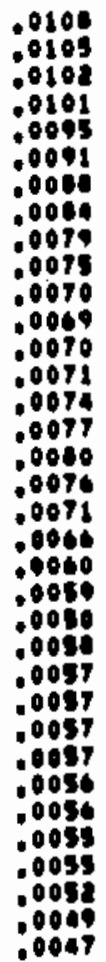 & 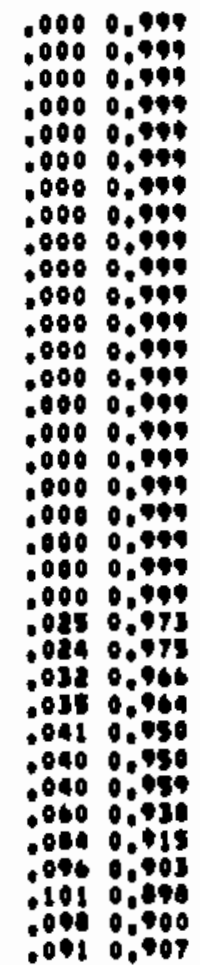 & $\begin{array}{l}0,00 \\
0.00 \\
0.00 \\
0.00 \\
0.00 \\
0.00 \\
0.00\end{array}$ & 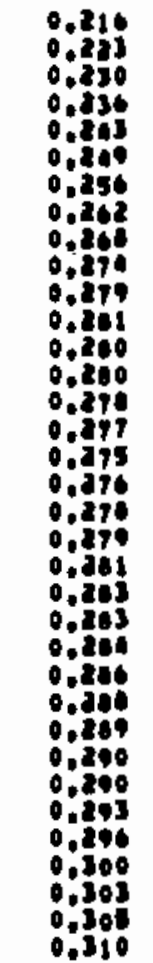 & $\begin{array}{l}.000 \\
0000 \\
0000 \\
0000 \\
0000 \\
0000 \\
0000 \\
0000 \\
0000 \\
0000 \\
0000 \\
0000 \\
0000 \\
0000 \\
000 \\
0000 \\
0001 \\
0008 \\
0004 \\
0005 \\
000 \\
0008 \\
0009 \\
0011 \\
0013 \\
0015 \\
0017 \\
0019 \\
0021 \\
0081 \\
0011 \\
0021 \\
0018 \\
0013 \\
0031\end{array}$ & $\begin{array}{l}100 \\
100 \\
100 \\
00 \\
00 \\
00 \\
100 \\
100 \\
100 \\
00 \\
00 \\
00 \\
10 \\
10 \\
79 \\
78\end{array}$ & $\begin{array}{l}0,000 \\
0,000 \\
0,000 \\
0,000 \\
0,000 \\
0,000 \\
0,000 \\
0,000 \\
0,000 \\
0,0000 \\
0.0000 \\
0,0000 \\
0,0000 \\
0,000 \\
0.000 \\
0.0000 \\
0.000\end{array}$ & $\begin{array}{l}00 \\
01 \\
01 \\
01 \\
01 \\
01 \\
01 \\
03 \\
03 \\
03 \\
03 \\
03\end{array}$ & 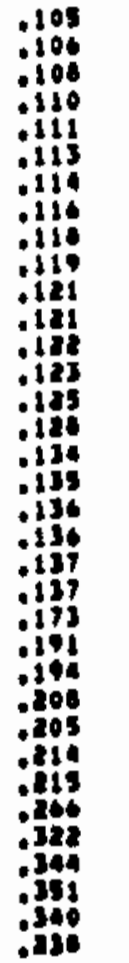 & $\begin{array}{l}11 \\
11 \\
11 \\
12 \\
12 \\
12 \\
12 \\
12 \\
12 \\
12 \\
12 \\
12\end{array}$ \\
\hline
\end{tabular}


TABLE A.15. FRAPCON-2 Prediction for $8 \times 8$ BWR with $9.4 \mathrm{~kW} / \mathrm{ft}$ for $20 \mathrm{GWd} / \mathrm{MTM}$, Ramp to $13.4 \mathrm{~kW} / \mathrm{ft}, \mathrm{P} / \mathrm{A}=1.3$

BUEL AVE AVE RAOIAL RAACTION OF CUMMULATIVE ROO

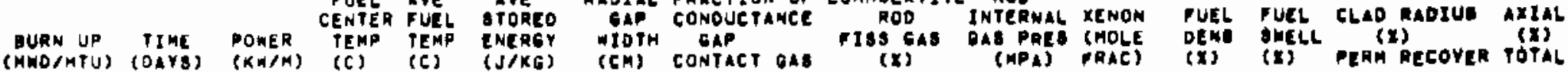


TABLE A.16. FRAPCON-2 Prediction for $15 \times 15 \mathrm{PWR}$ with $8.8 \mathrm{~kW} / \mathrm{ft}$ for 1 Day, Ramp to $12.6 \mathrm{~kW} / \mathrm{ft}$,
Constant Rod Average Power

TUEL AVE GVE RADIAL FRACTION OF CUMMULATIYE ROD

\begin{tabular}{|c|c|c|c|c|c|c|c|c|c|c|c|c|c|c|c|c|}
\hline $\begin{array}{l}\text { JRN UP } \\
\text { UD/MTU) }\end{array}$ & $\begin{array}{l}\text { TIME } \\
\text { (onyes }\end{array}$ & $\begin{array}{l}\text { POWER } \\
(K \in H / M)\end{array}$ & $\begin{array}{l}\text { FUEL } \\
\text { CENTEA } \\
\text { TEMP } \\
\text { (C) }\end{array}$ & $\begin{array}{l}\text { AVE } \\
\text { PUEL } \\
\text { TEMP } \\
\text { (C) }\end{array}$ & $\begin{array}{l}\text { AVE } \\
\text { ETOACD } \\
\text { CNERGY } \\
\text { (J/KES }\end{array}$ & $\begin{array}{l}\text { RADIAL } \\
\text { OAP } \\
\text { WOOTH } \\
\text { (CH) }\end{array}$ & $\begin{array}{l}\text { RACPID } \\
\text { CONOUCT } \\
\text { CONTACT }\end{array}$ & $\begin{array}{l}\text { DN OF } \\
\text { TANCE } \\
\text { T GAB }\end{array}$ & $\begin{array}{c}\text { CUMMULATIYE } \\
\text { ROD } \\
\text { FaB GAS } \\
\text { (X) }\end{array}$ & $\begin{array}{l}\text { ROD } \\
\text { NTERNAL } \\
\text { AO FRES } \\
\text { (MPA) }\end{array}$ & & $\begin{array}{l}\text { PUEL } \\
\text { Den } \\
(x)\end{array}$ & $\begin{array}{l}\text { FUEL } \\
\text { BRELL } \\
\text { (x) }\end{array}$ & $\begin{array}{c}\text { CLAO } \\
\text { PERM }\end{array}$ & & $\begin{array}{l}\operatorname{AxIAL} \\
\text { TOT: } \\
\text { TOT }\end{array}$ \\
\hline $\begin{array}{r}0 \\
0 \\
0 \\
1 \\
2 \\
3 \\
4 \\
5 \\
7 \\
19 \\
43 \\
49 \\
56 \\
112\end{array}$ & $\begin{array}{l}0.01 \\
0.03 \\
0.06 \\
0.09 \\
0.12 \\
0.15 \\
0.18 \\
0.012 \\
0.04 \\
0.100 \\
1.00\end{array}$ & $\begin{array}{l}0.01 \\
3.61 \\
7.21 \\
10.62 \\
14.45 \\
18.04 \\
21.64 \\
25.45 \\
20.74 \\
30.74 \\
20.74 \\
39.26 \\
40.95 \\
40.05\end{array}$ & $\begin{array}{l}274 \\
390 \\
940 \\
543 \\
649 \\
700 \\
676 \\
997 \\
1110 \\
1110 \\
1120 \\
1392 \\
1510 \\
1512\end{array}$ & $\begin{array}{l}279 \\
321 \\
369 \\
220 \\
473 \\
528 \\
585 \\
643 \\
701 \\
701 \\
702 \\
1010 \\
003 \\
904\end{array}$ & 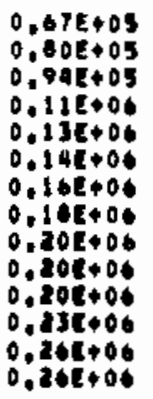 & $\begin{array}{l}.0086 \\
00005 \\
00082 \\
00079 \\
00075 \\
00072 \\
00069 \\
00065 \\
00060 \\
00060 \\
00062 \\
.0053 \\
.0047 \\
.0048\end{array}$ & 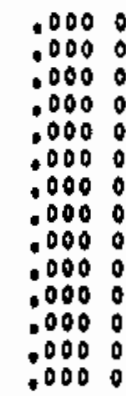 & $\begin{array}{l}0.999 \\
0.999 \\
0.999 \\
0.999 \\
0.999 \\
0.999 \\
0.999 \\
0.999 \\
0.999 \\
0.999 \\
0.999 \\
0.999 \\
0.999 \\
0.999\end{array}$ & $\begin{array}{l}0 . \\
0 . \\
0 . \\
0 . \\
0 . \\
0 .\end{array}$ & 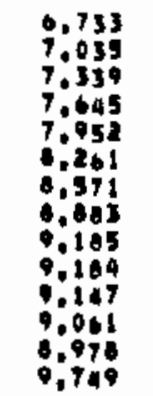 & $\begin{array}{l}.000 \\
.000 \\
.000 \\
0000 \\
.000 \\
.000 \\
.000 \\
.000 \\
.000 \\
.000 \\
.000 \\
.000 \\
.000\end{array}$ & $\begin{array}{l}000 \\
0000 \\
000 \\
000 \\
000 \\
000 \\
000 \\
000 \\
000 \\
000 \\
028 \\
0 \$ 0 \\
039 \\
071\end{array}$ & & $\begin{array}{l}0000 \\
0000 \\
000 \\
0001 \\
0001 \\
0001 \\
001 \\
002 \\
002 \\
000 \\
007 \\
000 \\
000 \\
010\end{array}$ & $\begin{array}{l}0 \\
0 \\
0 \\
0 \\
0 \\
0 \\
\vdots 1 \\
\vdots 1 \\
11 \\
11 \\
11\end{array}$ & $\begin{array}{ll}1 & 0 \\
11 \\
11 \\
11 \\
11 \\
11 \\
11 \\
11 \\
12 \\
12 \\
12 \\
12 \\
12\end{array}$ \\
\hline
\end{tabular}


TABLE A.17. FRAPCON-2 Prediction for $15 \times 15$ PWR with $8.8 \mathrm{~kW} / \mathrm{ft}$ for $10 \mathrm{GWd} / \mathrm{MTM}$, Ramp to $12.6 \mathrm{~kW} / \mathrm{ft}$, Constant Rod Average Power

\begin{tabular}{|c|c|c|c|c|c|c|c|c|c|c|c|c|c|c|c|c|}
\hline S UP & $\begin{array}{l}\text { TIME } \\
\text { (DAYS) }\end{array}$ & $\begin{array}{l}\text { POWER } \\
(x \sim M)\end{array}$ & $\begin{array}{l}\text { FUEL } \\
\text { CENTER } \\
\text { TEMP } \\
\text { (C) }\end{array}$ & $\begin{array}{l}\text { AVE } \\
\text { TUEL } \\
\text { TEMP } \\
\text { (c) }\end{array}$ & $\begin{array}{l}\text { AVE } \\
\text { STOAEO } \\
\text { EYERGY } \\
(J / K G)\end{array}$ & $\begin{array}{l}\text { RADIAL } \\
\text { OAP } \\
\text { WIOTH } \\
\text { (CH) }\end{array}$ & $\begin{array}{l}\text { FRACTIO } \\
\text { CONOUCT } \\
\text { GAP } \\
\text { CONTACT }\end{array}$ & 643 & $\begin{array}{c}\text { CUMHULATIV } \\
\text { FOD } \\
\text { FIS8 OAS } \\
\text { (X) }\end{array}$ & $\begin{array}{l}\text { ROD } \\
\text { INTERNAL } \\
\text { GAB PRES } \\
\text { (MPA) }\end{array}$ & $\begin{array}{l}\text { XENON } \\
\text { (MOLE } \\
\text { PRAC) }\end{array}$ & $\begin{array}{l}\text { FUEL } \\
\text { DENS } \\
(x)\end{array}$ & $\begin{array}{l}\text { UEL } \\
\text { BWELL } \\
(x)\end{array}$ & $\begin{array}{c}\text { CLAO } \\
\text { TER }\end{array}$ & & 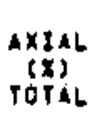 \\
\hline $\begin{array}{r}0 \\
0 \\
0 \\
1 \\
2 \\
3 \\
4 \\
5 \\
7 \\
19 \\
43 \\
48 \\
53 \\
92 \\
237 \\
490 \\
965 \\
1936 \\
2906 \\
3876 \\
4807 \\
6059 \\
7272 \\
8485 \\
9698 \\
10426 \\
10432 \\
10439 \\
10494\end{array}$ & $\begin{array}{l}0.01 \\
0.03 \\
0.06 \\
0.00 \\
0.12 \\
0.15 \\
0.18 \\
0.21 \\
0.20 \\
0.50 \\
1.00 \\
1.10 \\
1.20 \\
2.00 \\
5.00 \\
10.00 \\
20.00 \\
00.00 \\
60.00 \\
80.00 \\
190.00 \\
125.00 \\
150.00 \\
175.00 \\
200.00 \\
215.00 \\
215.10 \\
215.20\end{array}$ & $\begin{array}{r}0.01 \\
3.61 \\
7.21 \\
10.82 \\
14.43 \\
18.04 \\
21.64 \\
25.25 \\
28.74 \\
28.74 \\
28.74 \\
28.74 \\
78.74 \\
20.74 \\
24.74 \\
28.74 \\
28.74 \\
28.74 \\
78.74 \\
28.74 \\
28.74 \\
29.74 \\
28.74 \\
28.74 \\
28.74 \\
28.74 \\
35.26 \\
40.95\end{array}$ & $\begin{array}{l}274 \\
358 \\
440 \\
545 \\
609 \\
980 \\
876 \\
997 \\
1110 \\
1110 \\
1120 \\
1120 \\
1120 \\
1121 \\
1129 \\
1124 \\
1119 \\
1104 \\
1078 \\
1054 \\
1032 \\
1009 \\
993 \\
994 \\
979 \\
979 \\
9178 \\
11337 \\
1339 \\
1306\end{array}$ & $\begin{array}{l}274 \\
321 \\
309 \\
420 \\
473 \\
528 \\
505 \\
643 \\
701 \\
701 \\
702 \\
701 \\
702 \\
702 \\
103 \\
703 \\
701 \\
695 \\
685 \\
675 \\
666 \\
657 \\
650 \\
645 \\
643 \\
602 \\
732 \\
609 \\
708\end{array}$ & 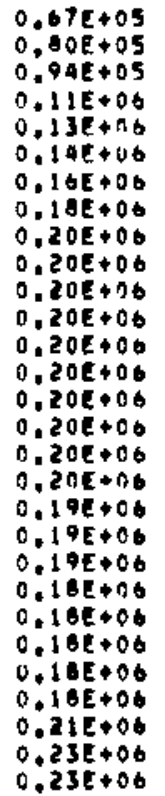 & 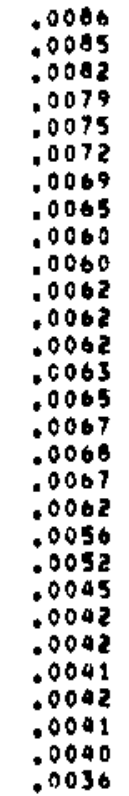 & 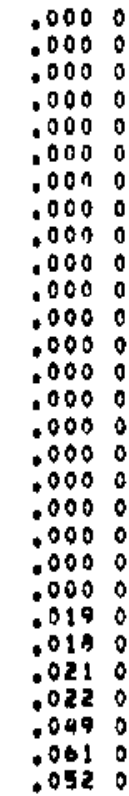 & $\begin{array}{l}970 \\
978 \\
950 \\
970\end{array}$ & $\begin{array}{l}0.00 \\
0.00 \\
0.00 \\
0.00 \\
0.00 \\
0.00 \\
0.00 \\
0.00 \\
0.00 \\
0.00\end{array}$ & $\begin{array}{l}6.733 \\
1.035 \\
7.335\end{array}$ & 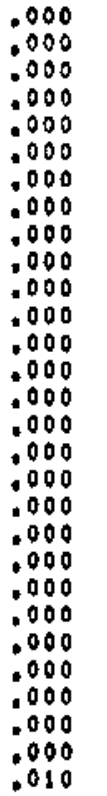 & $\begin{array}{l}000 \\
0000 \\
000 \\
0000 \\
000 \\
000 \\
000 \\
000 \\
000 \\
000 \\
000 \\
028 \\
031 \\
034 \\
098 \\
120 \\
186 \\
1238 \\
257 \\
258 \\
1258\end{array}$ & $\begin{array}{l}0,0 \\
0,0 \\
0: 0 \\
0,0 \\
0.0 \\
0: 0 \\
0: 0 \\
0,0 \\
0,0 \\
0,0 \\
0,0 \\
0,0 \\
0,0 \\
0: 0 \\
0: 0 \\
0,0 \\
0,0 \\
0: 1 \\
0.1\end{array}$ & 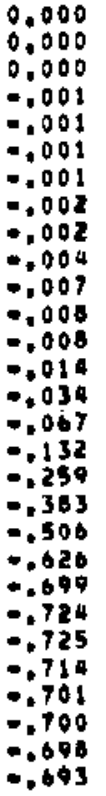 & 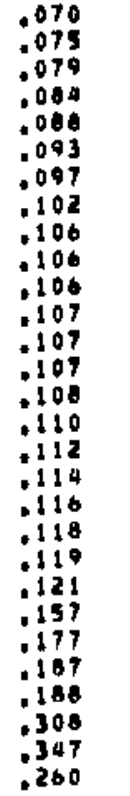 & $\begin{array}{l}0.10 \\
0.11 \\
0.11 \\
0.11 \\
0.11 \\
0.11 \\
0.11 \\
0.11 \\
0.12 \\
0.12 \\
0.12 \\
0.12 \\
0.12 \\
0.12 \\
0.13 \\
0.13 \\
0.19 \\
0.12 \\
0.15 \\
0.15 \\
0.15 \\
0.15 \\
0.15 \\
0.15 \\
0.17 \\
0.131 \\
0.40 \\
0.35\end{array}$ \\
\hline
\end{tabular}


TABLE A.18. FRAPCON-2 Prediction for $15 \times 15$ PWR with $8.8 \mathrm{~kW} / \mathrm{ft}$ for $20 \mathrm{GWd} / \mathrm{MTM}$, Ramp to $12.6 \mathrm{~kW} / \mathrm{ft}$, Constant Rod Average Power

PUEL AVE AVE RADIAL FRACTION OF CUMMULATIVE hOO

\begin{tabular}{|c|c|c|c|c|c|c|c|c|c|c|c|c|c|c|c|c|}
\hline $\begin{array}{l}\text { BURN UP } \\
\text { (ANO/MTU) }\end{array}$ & $\begin{array}{c}\text { TIME } \\
\text { (DAYS) }\end{array}$ & $\begin{array}{l}\text { POWER } \\
(K W / m)\end{array}$ & $\begin{array}{l}\text { FUEL } \\
\text { CENTER } \\
\text { PEMP } \\
\text { (C) }\end{array}$ & $\begin{array}{l}\text { AVE } \\
\text { PUEL } \\
\text { TEPP } \\
\text { (C) }\end{array}$ & $\begin{array}{l}\text { AVE } \\
\text { PTORED } \\
\text { ENEREY } \\
\text { (J/KG) }\end{array}$ & $\begin{array}{l}\text { RADIAL } \\
\text { OAP } \\
\text { WIOTH } \\
\text { (CM) }\end{array}$ & $\begin{array}{l}\text { FRACTIO } \\
\text { CONDUCT } \\
\text { OAP } \\
\text { CONTAC? }\end{array}$ & $\begin{array}{l}\text { ION OF } \\
\text { ETANCE } \\
\text { CT GAO }\end{array}$ & $\begin{array}{c}\text { CUMMULATIVE } \\
\text { ROD } \\
\text { FIOA GAO } \\
\text { [KS }\end{array}$ & $\begin{array}{l}\text { AOO } \\
\text { INTERNAL } \\
\text { GAS PRES } \\
\text { (MPA) }\end{array}$ & $\begin{array}{l}\text { XENON } \\
\text { (MOLE } \\
\text { FRAC) }\end{array}$ & & $\begin{array}{l}\text { UEL } \\
\text { AWELL } \\
(x)\end{array}$ & $\begin{array}{l}\text { CLAD } \\
\text { PEMM }\end{array}$ & $\begin{array}{l}\text { ADIUS } \\
\text { ECOVER }\end{array}$ & $\begin{array}{c}A \times I A L \\
(X) \\
T O T A L\end{array}$ \\
\hline $\begin{array}{r}0 \\
0 \\
0 \\
1 \\
2 \\
3 \\
4 \\
5 \\
7 \\
19 \\
43 \\
48 \\
53 \\
92 \\
237 \\
980 \\
965 \\
1936 \\
2906 \\
3676 \\
9847 \\
6059 \\
7272 \\
8085 \\
9698 \\
10911 \\
12124 \\
13337 \\
19550 \\
15763 \\
16976 \\
18169 \\
19401 \\
20614 \\
20857 \\
20863 \\
20870 \\
20925\end{array}$ & 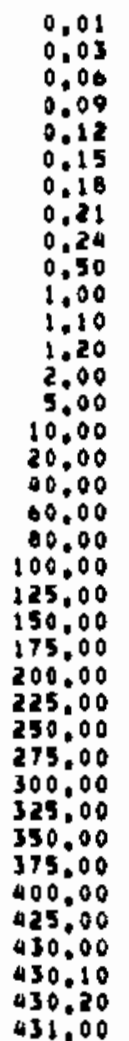 & 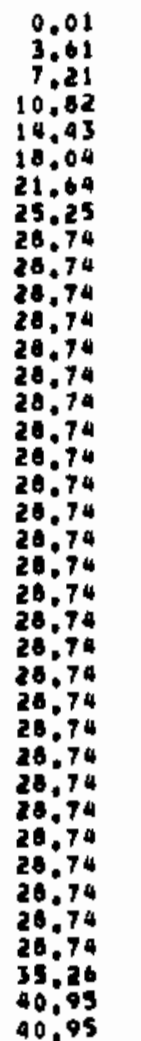 & $\begin{array}{l}274 \\
358 \\
440 \\
545 \\
649 \\
760 \\
676 \\
907 \\
1118 \\
1118 \\
1120 \\
1120 \\
1120 \\
1121 \\
1124 \\
1124 \\
1119 \\
1104 \\
1078 \\
1054 \\
1032 \\
1009 \\
993 \\
904 \\
979 \\
975 \\
972 \\
972 \\
971 \\
970 \\
969 \\
969 \\
969 \\
970 \\
972 \\
1167 \\
1319 \\
1306\end{array}$ & $\begin{array}{l}274 \\
321 \\
369 \\
420 \\
473 \\
528 \\
505 \\
643 \\
701 \\
701 \\
702 \\
701 \\
702 \\
702 \\
703 \\
703 \\
701 \\
695 \\
685 \\
675 \\
606 \\
657 \\
650 \\
645 \\
643 \\
641 \\
640 \\
640 \\
641 \\
641 \\
641 \\
641 \\
642 \\
642 \\
644 \\
733 \\
610 \\
600\end{array}$ & 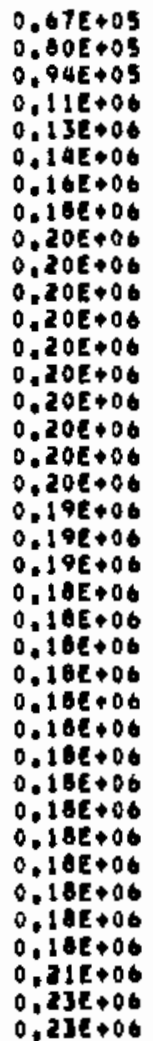 & $\begin{array}{l}.0086 \\
.0085 \\
.0002 \\
00079 \\
00075 \\
00072 \\
00069 \\
00065 \\
00060 \\
00060 \\
00062 \\
00062 \\
00063 \\
00065 \\
00067 \\
00068 \\
00067 \\
00062 \\
00056 \\
00052 \\
00045 \\
00042 \\
00042 \\
00041 \\
00041 \\
.0041 \\
00041 \\
00036 \\
00041 \\
00041 \\
00041 \\
00040 \\
00037 \\
00041 \\
00040 \\
00039 \\
00035\end{array}$ & 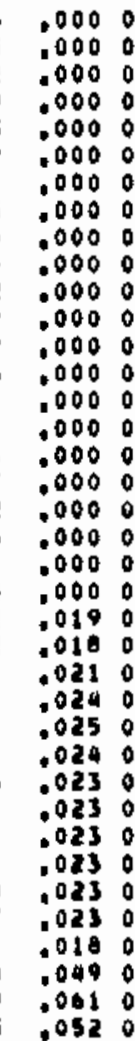 & $\begin{array}{l}0,999 \\
0,999 \\
0,999 \\
0,999 \\
0,990 \\
0,999 \\
0,999 \\
0,999 \\
0,999 \\
0,999 \\
0,999 \\
0,999 \\
0,999 \\
0,999 \\
0,999 \\
0,999 \\
0,999 \\
0,999 \\
0,999 \\
0,999 \\
0.999 \\
0,999 \\
0,900 \\
0,961 \\
0,979 \\
0,975 \\
0,974 \\
0,975 \\
0,976 \\
0,976 \\
0,976 \\
0,976 \\
0,976 \\
0,976 \\
0,900 \\
0,950 \\
0,938 \\
0,947\end{array}$ & $\begin{array}{l}0.00 \\
0.00 \\
0.00 \\
0.00 \\
0.00 \\
0.00 \\
0.00 \\
0.00 \\
0.00 \\
0.00 \\
0.00 \\
0.00 \\
0.01 \\
0.01 \\
0.01 \\
0.02 \\
0.02 \\
0.03 \\
0.04 \\
0.04 \\
0.05 \\
0.06 \\
0.06 \\
0.07 \\
0.09 \\
0.10 \\
0.11 \\
0.12 \\
0.13 \\
0.14 \\
0.15 \\
0.17 \\
0.10 \\
0.19 \\
0.20 \\
0.20 \\
0.20 \\
0.25\end{array}$ & $\begin{array}{l}0 \\
5 \\
2 \\
1 \\
1 \\
3 \\
5 \\
7 \\
7\end{array}$ & $\begin{array}{l}.000 \\
0000 \\
0000 \\
0000 \\
0000 \\
000 \\
0000 \\
0000 \\
0000 \\
0000 \\
0000 \\
0000 \\
0000 \\
0000 \\
0000 \\
0000 \\
0000 \\
0000 \\
0000 \\
0000 \\
0000 \\
0000 \\
0000 \\
0000 \\
0000 \\
0000 \\
0001 \\
0001 \\
0001 \\
0001 \\
0001 \\
0001 \\
0002 \\
0002 \\
0002 \\
0002 \\
0002 \\
0002\end{array}$ & 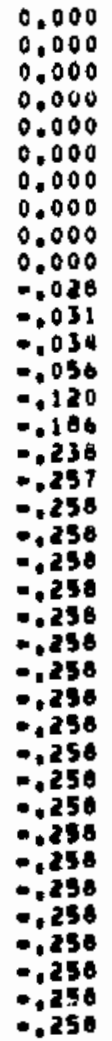 & 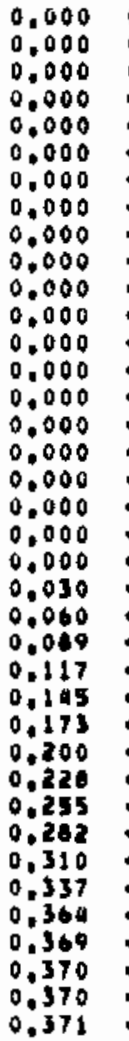 & 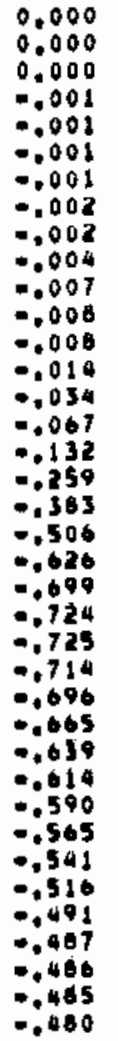 & 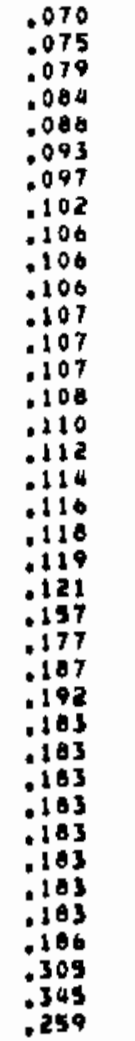 & $\begin{array}{l}0.10 \\
0.10 \\
0.11 \\
0.11 \\
0.11 \\
0.11 \\
0.11 \\
0.11 \\
0.11 \\
0.12 \\
0.12 \\
0.12 \\
0.12 \\
0.12 \\
0.12 \\
0.13 \\
0.13 \\
0.14 \\
0.14 \\
0.15 \\
0.15 \\
0.15 \\
0.14 \\
0.15 \\
0.17 \\
0.14 \\
0.22 \\
0.24 \\
0.27 \\
0.29 \\
0.32 \\
0.34 \\
0.37 \\
0.39 \\
0.40 \\
0.52 \\
0.61 \\
0.59\end{array}$ \\
\hline
\end{tabular}


TABLE A.19. GAPCON-2 Prediction for $15 \times 15$ with $12.6 \mathrm{~kW} / \mathrm{ft}$ to $20 \mathrm{GWd} / \mathrm{MTM}, \mathrm{P} / \mathrm{A}=1.2$

PUEL AVE aVe GAOTAL PAACTION OF CUMMULATIVE ROD

\begin{tabular}{|c|c|c|c|c|c|c|c|c|c|c|c|c|c|c|c|c|}
\hline URN UP & (Dime & $\begin{array}{l}\text { POWER } \\
(M=/ M)\end{array}$ & $\begin{array}{l}\text { CENFER } \\
\text { TEMP } \\
\text { (C) }\end{array}$ & $\begin{array}{l}\text { RUE } \\
\text { TEM } \\
\text { (C) }\end{array}$ & $\begin{array}{l}\text { AVE } \\
\text { ERORED } \\
\text { CNEASY } \\
\text { (J/KO) }\end{array}$ & $\begin{array}{c}\text { ROIAL } \\
\text { OAP } \\
\text { TIDYH } \\
(E H)\end{array}$ & $\begin{array}{l}\text { CONOUC } \\
\text { OAP } \\
\text { CONTAE }\end{array}$ & TAMCE & $\begin{array}{c}\text { (x) } \\
\text { ROD } \\
\text { OAO }\end{array}$ & $\begin{array}{c}\text { INTERMAL } \\
\text { GAS PREA } \\
\text { (MPAS }\end{array}$ & $\begin{array}{l}\text { XCNON } \\
\text { (HOLE } \\
\text { PRAC) }\end{array}$ & $\begin{array}{l}\text { FUEL } \\
\text { OENS } \\
\text { (I) }\end{array}$ & $\begin{array}{l}\text { Fuet } \\
\text { fweth } \\
(x)\end{array}$ & $\begin{array}{l}\text { CLAD } \\
\text { PERM } \\
\text { PE }\end{array}$ & & $\begin{array}{c}A x I \\
(x) \\
\text { roti }\end{array}$ \\
\hline $\begin{array}{l}1970 \\
3956 \\
3034 \\
7912 \\
9090 \\
11868 \\
13006 \\
15024 \\
17004 \\
19780\end{array}$ & 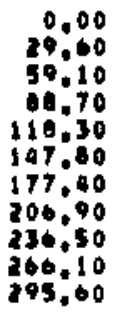 & $\begin{array}{l}41.33 \\
41.33 \\
41.33 \\
41.33 \\
91.33 \\
41.33 \\
91.33 \\
41.33 \\
41.33 \\
41.33 \\
41.33\end{array}$ & $\begin{array}{l}1050 \\
1528 \\
1516 \\
1516 \\
1516 \\
1517 \\
1518 \\
1519 \\
1087 \\
1455 \\
1907\end{array}$ & $\begin{array}{l}1039 \\
945 \\
937 \\
935 \\
935 \\
936 \\
936 \\
937 \\
913 \\
887 \\
882\end{array}$ & 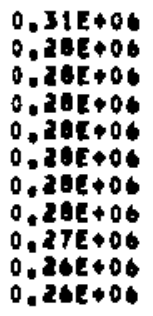 & $\begin{array}{l}.0033 \\
.00211 \\
.0019 \\
.0020 \\
.0019 \\
.0019 \\
.0019 \\
.0019 \\
.0016 \\
.0013 \\
.0012\end{array}$ & $\begin{array}{l}.000 \\
.000 \\
.000 \\
.000 \\
.000 \\
000 \\
0000 \\
.000 \\
.000 \\
.000 \\
.000\end{array}$ & $\begin{array}{l}0,980 \\
0,991 \\
0,991 \\
0,991 \\
0.991 \\
0,991 \\
0,991 \\
0,991 \\
0,993 \\
0,993 \\
1.000\end{array}$ & $\begin{array}{l}0 . \\
0 . \\
0 . \\
0 .\end{array}$ & 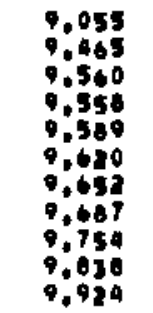 & $\begin{array}{l}.000 \\
.001 \\
.003 \\
.004 \\
.006 \\
.008 \\
.011 \\
.013 \\
.016 \\
.016 \\
.020\end{array}$ & $\begin{array}{l}0.000 \\
=.004 \\
=004 \\
=004 \\
=004 \\
=004 \\
=004 \\
=004 \\
=004 \\
=004 \\
=.004\end{array}$ & $\begin{array}{l}0 \\
0 \\
0 \\
0 \\
0 \\
0 \\
0 \\
0 \\
0\end{array}$ & & & $\begin{array}{l}- \\
-\end{array}$ \\
\hline
\end{tabular}


TABLE A.20. GAPCON-2 Prediction for $15 \times 15$ with $8.8 \mathrm{~kW} / \mathrm{ft}$ for 20 GWd/MTM Ramp to $12.6 \mathrm{~kW} / \mathrm{ft}, P / A=1.2$

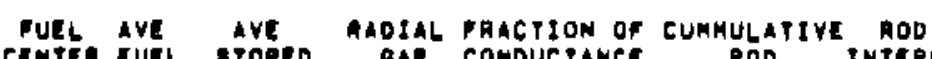

BUPA UP TIME POMER CEMTEN FUTL STORED GAP CONDUCPANCE ROA

TIBB OAO INTERNAL XENON FUEL FUEL CLAO RADIUS AXIAL

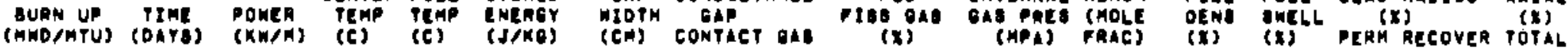

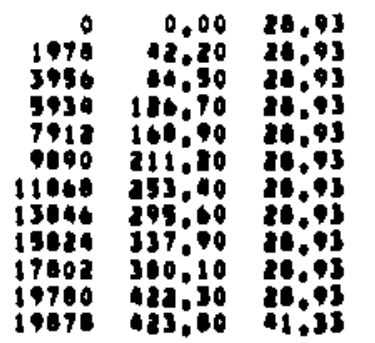

\begin{tabular}{|c|c|c|c|c|c|}
\hline $\begin{array}{l}1230 \\
1142 \\
1133 \\
1133 \\
1133 \\
1133 \\
1139 \\
1134 \\
1113 \\
1098 \\
1497 \\
1496\end{array}$ & $\begin{array}{l}647 \\
779 \\
772 \\
778 \\
772 \\
772 \\
773 \\
773 \\
710 \\
700 \\
609\end{array}$ & 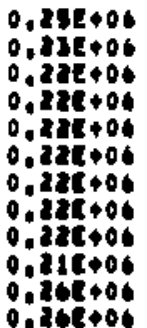 & $\begin{array}{l}.0050 \\
.0035 \\
.0034 \\
00034 \\
00034 \\
.0034 \\
00033 \\
0033 \\
0030 \\
00086 \\
0012\end{array}$ & $\begin{array}{l}.000 \\
0000 \\
0000 \\
0000 \\
0000 \\
000 \\
0000 \\
0000 \\
0000 \\
0000 \\
0000 \\
0000\end{array}$ & $\begin{array}{l}0.997 \\
0.997 \\
0.997 \\
0.997 \\
0.997 \\
0.997 \\
0.997 \\
0.997 \\
0.997 \\
1.000 \\
1.005 \\
1.000\end{array}$ \\
\hline
\end{tabular}

\begin{tabular}{|c|c|c|c|c|c|}
\hline $\begin{array}{l}18 \\
01 \\
06\end{array}$ & $\begin{array}{l}.000 \\
.000 \\
0000 \\
0001 \\
0001 \\
.002 \\
.004 \\
005 \\
0007 \\
0009 \\
0017 \\
017\end{array}$ & 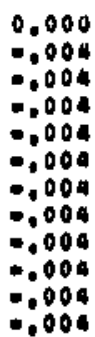 & $\begin{array}{l}0.000 \\
0.000 \\
0.000 \\
0.000 \\
0.0000 \\
0.000 \\
0.000 \\
0.000 \\
0.001 \\
0.002 \\
0.001 \\
0.003\end{array}$ & $\begin{array}{l}0,000 \\
0,000 \\
0,000 \\
0,000 \\
0,000 \\
0,0000 \\
0,0000 \\
0,000 \\
0,000 \\
0,000 \\
0,000 \\
0,000\end{array}$ & $\begin{array}{l}.001 \\
001 \\
0001 \\
001 \\
0001 \\
001 \\
001 \\
001 \\
001 \\
001 \\
001 \\
.001\end{array}$ \\
\hline
\end{tabular}


NUREG/CR-2369

PNL-4059

\section{DISTRIBUTION}

No. of

Copies

OFFSITE

A. A. Churm

DOE Patent 0ivision

$9800 \mathrm{~S}$. Cass Avenue

Argonne, IL 60439

400 U.S. Nuclear Regulatory Commission

Division of Technical Information and Document Control

7920 Norfolk Avenue

Bethesda, MD 20014

2 O0E Technical Information Center

C. Berl inger

Chief, Core Performance Branch

Division of Systems Safety

U.S. Nuclear Regulatory Commission

Washington, DC 20555

G. P. Marino

Fuel Behavior Research Branch

Division of Reactor Safety

Research

Office of Nuclear Regulatory

Research

U.S. Nuclear Regulatory Commission

Washington, DC 20555

R. 0 . Meyer

Core Performance Branch

Division of Systems Safety

U.S. Nuclear Regulatory Commission

Washington, DC 20555
No. of

Copies

H. H. Scott

Fuel Behavior Research Branch

Division of Reactor Safety

Research

office of Nuclear Regulatory

Research

U.S. Nuclear Regulatory Commission

Washington, DC 20555

M. Tokar

Reactor Fuels Section

Core Performance Branch

Department of Licensing

U.S. Nuclear Regulatory

Commission

Washington, DC 20555

10 J. C. Voglewede

Reactor Fuels Section

Core Performance Branch

Division of Systems Safety

U.S. Nuclear Regulatory Commission

Washington, DC 20555

G. A. Berna

EG\&G Idaho, Inc.

550 2nd St.

Idaho Falls, ID 83401

ONSITE

33 Pacific Northwest Laboratory

W. D. Bennett

C. E. Beyer

E. R. Bradley

M. E. Cunningham 
No. of

Copies

S. K. Edler

M. D. Freshley

E. R. Gilbert (5)

R. J. Guenther

C. R. Hann

D. D. Lanning

C. L. Mohr

F. E. Panisko (5)

W. N. Rausch (5)

R. E. Williford

Technical Information YO(5)

Publishing Coordination (2) 


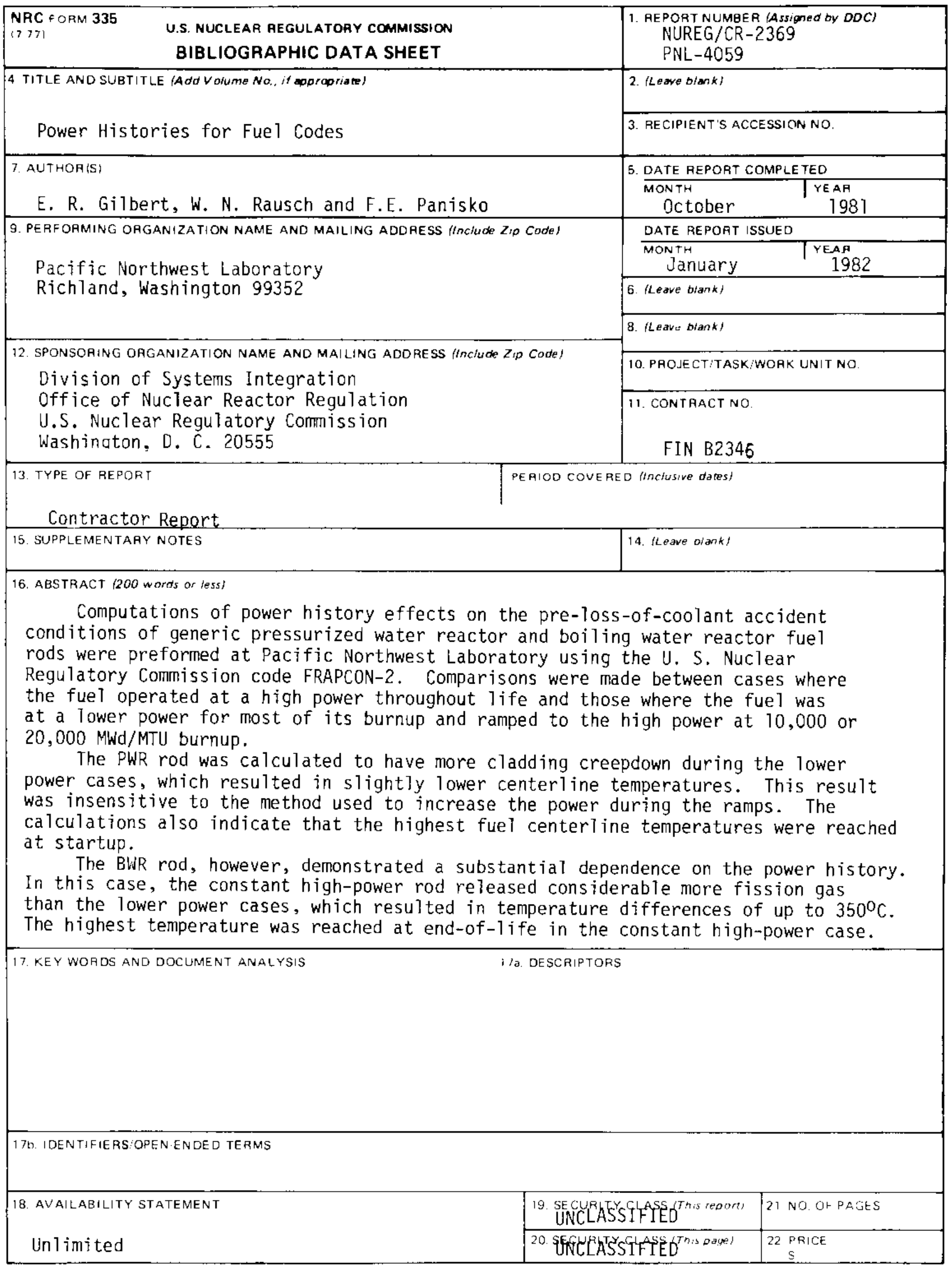


